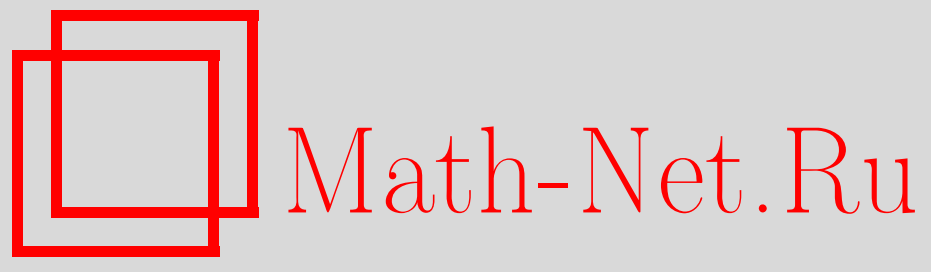

И. Х. Сабитов, Изометричные поверхности с общей средней кривизной и проблема пар Бонне, Матем. сб., 2012, том 203, номер 1, 115-158

DOI: https://doi.org/10.4213/sm7706

Использование Общероссийского математического портала Math-Net.Ru подразумевает, что вы прочитали и согласны с пользовательским соглашением http://www . mathnet.ru/rus/agreement

Параметры загрузки:

IP: 34.239 .49 .27

26 апреля 2023 г., 18:05:37

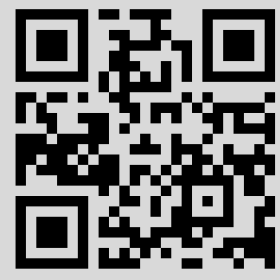




\title{
И. Х. Сабитов \\ Изометричные поверхности с общей средней кривизной и проблема пар Бонне
}

\begin{abstract}
В работе простыми методами передоказываются, а иногда и уточняются основные теоремы об изометричных поверхностях с общей средней кривизной, которые принято называть парами Бонне. Рассмотрения ведутся в предположении минимально допустимой гладкости рассматриваемых объектов и приводятся некоторые необходимые или достаточные признаки несуществования среди компактных поверхностей пар Бонне с общей непостоянной средней кривизной.
\end{abstract}

Библиография: 26 названий.

Ключевые слова: поверхности, изометрия, средняя кривизна, инвариантность.

\section{§1. Введение}

Вопрос о поверхностях с общей метрикой и общей средней кривизной впервые рассмотрен Бонне в работе [1], в которой он сформулирован как проблема существования изометрического отображения одной поверхности на другую с сохранением главных кривизн. Бонне показал, что в общем случае поверхность даже локально определяется однозначно своей метрикой и средней кривизной, но в частных случаях могут существовать и неконгруентные изометричные поверхности с совпадением средних кривизн в соответствующих по изометрии точках. Локальному описанию поверхностей с заданной средней кривизной и их изгибаниям посвящено значительное число работ (см., например, [2]-[7]; более подробный список литературы можно найти, например, в статье [8] или в книге [9]). В глобальной постановке для проблемы существования пары изометричных поверхностей с одинаковой средней кривизной (которые и называются парами Бонне) первые результаты получены в работах [10] и [11]. В [10] доказано, что компактные поверхности рода $p=0$ однозначно определены своими метрикой и средней кривизной (т.е. среди них нет ни одной пары Бонне), а среди поверхностей рода $p=1$ для данной метрики и данной непостоянной средней кривизны может существовать не более одной пары Бонне (т.е. не существует троек Бонне). В [11] этот результат работы [10] обобщен на поверхности произвольного рода, но вопрос о фактическом существовании/несуществовании такой пары остался открытым.

Работа выполнена при поддержке Российского фонда фундаментальных исследований (грант № 09-01-00179) и Министерства образования РФ (грант № РНП 2.1.1.3704).

(С) И.Х. САвитов, 2012 
В статье Бонне [1] все рассмотрения ведутся для комплексно-аналитических поверхностей и включают такие объекты, как мнимые линии, и поэтому эта работа довольно трудна для понимания, тем более, что ряд ее выводов не имеет места в вещественнозначной ситуации (см. [2]). В самой работе [2] рассматриваемые поверхности уже предполагаются вещественно-аналитическими. В дальнейших работах классы гладкости поверхностей, как правило, не уточняются, но обычно неявно предполагается их априорная $C^{\infty}$-гладкость (см., например, [8]) и лишь в некоторых случаях уточняется, начиная с какого класса гладкости верны доказываемые утверждения (см., например, [9; с. 22]). Поэтому в результате остается неясным, например, в каком классе гладкости и в каком смысле "в общем" метрика и средняя кривизна определяют поверхность однозначно, а при каких исходных условиях гладкости поверхности семейства Бонне на самом деле являются аналитическими.

В настоящей статье мы сначала выведем формулу, позволяющую явно выразить коэффициенты второй квадратичной формы поверхности через ее метрику и среднюю кривизну и дающую точный смысл утверждению, что "в общем" метрика и средняя кривизна определяют поверхность однозначно. Рассмотрев случаи, когда такой однозначности нет, мы дадим новые доказательства ряда известных теорем о таких поверхностях и уточним доказательства некоторых из них, причем в предположении изначально минимально допустимой гладкости рассматриваемых поверхностей и функций. В заключение мы докажем, что среди компактных поверхностей гладкости класса $C^{2}$ и рода $p=0$ нет пар Бонне и в том же классе гладкости для поверхностей с непостоянной средней кривизной и произвольного рода $p>0$ нет троек Бонне. Что касается пар Бонне, то для поверхностей с непостоянной средней кривизной и гладкости класса $C^{4}$ мы дадим ряд достаточных условий их несуществования.

\section{§ 2. Локальные свойства дифференциалов Хопфа}

Пусть метрика поверхности $S \in C^{3}$ в $\mathbb{R}^{3}$ записана в изотермических координатах $(\xi, \eta)$ в виде

$$
d s^{2}=\Lambda^{2}(\xi, \eta)\left(d \xi^{2}+d \eta^{2}\right)
$$

и пусть

$$
K=\frac{L N-M^{2}}{\Lambda^{4}}, \quad H=\frac{L+N}{2 \Lambda^{2}}
$$

- соответственно гауссова и средняя кривизны поверхности, а $L, M, N$ - коэффициенты ее второй квадратичной формы. Положим $\zeta=\xi+i \eta$ и введем операции дифференцирования

$$
\frac{\partial}{\partial \bar{\zeta}}=\frac{\partial}{\partial \xi}+i \frac{\partial}{\partial \eta}, \quad \frac{\partial}{\partial \zeta}=\frac{\partial}{\partial \xi}-i \frac{\partial}{\partial \eta} .
$$

Обозначим

$$
f=2 \Lambda^{2} \frac{\partial H}{\partial \zeta}, \quad g=4\left(H^{2}-K\right) \Lambda^{4}, \quad \Omega=(L-N)-2 i M .
$$


Непосредственно проверяется, что во введенных обозначениях уравнения Гаусса и Петерсона-Кодацци имеют соответственно вид

$$
\begin{aligned}
& \Omega \bar{\Omega}=g, \\
& \frac{\partial \Omega}{\partial \bar{\zeta}}=f .
\end{aligned}
$$

Квадратичный дифференциал $\Omega d \zeta^{2}$ называется дифференииалом Хопфа. При замене изотермических координат $\zeta=\zeta(\widetilde{\zeta})$ он изменяется по закону

$$
\Omega(\zeta)(d \zeta)^{2}=\widetilde{\Omega}(\widetilde{\zeta})(d \widetilde{\zeta})^{2}, \quad \text { где } \widetilde{\Omega}(\widetilde{\zeta})=\Omega(\zeta(\widetilde{\zeta})) \zeta^{\prime 2}(\widetilde{\zeta})
$$

Далее нам часто будут встречаться инвариантные дифференциальные формы вида $F d \zeta^{m} d \bar{\zeta}^{n}$, будем называть их $F$-дифференииалами порядка $(m, n)$ или порядка $m+n$, а об их коэффициенте будем говорить как о коэффициенте $F$-формы. Например, введенные выше величины $f$ и $g$ не являются функциями точки, а являются коэффициентами инвариантных дифференциальных форм порядков $(2,1)$ и $(2,2)$ соответственно вида $f(\zeta) d \zeta^{2} d \bar{\zeta}$ и $g(\zeta) d \zeta^{2} d \bar{\zeta}^{2}$, изменяющимися при конформной замене координат $\zeta=\zeta(\widetilde{\zeta})$ по закону

$$
\widetilde{f}(\widetilde{\zeta})=f(\zeta(\widetilde{\zeta})) \zeta^{\prime 2}(\widetilde{\zeta}) \bar{\zeta}^{\prime}(\widetilde{\zeta}), \quad \widetilde{g}(\widetilde{\zeta})=g(\zeta(\widetilde{\zeta})) \zeta^{\prime 2}(\widetilde{\zeta}) \bar{\zeta}^{2}(\widetilde{\zeta})
$$

При известном коэффициенте $\Omega$ и известной средней кривизне $H$ коэффициенты второй квадратичной формы поверхности вычисляются по формулам

$$
L=H \Lambda^{2}+\frac{1}{2} \operatorname{Re} \Omega, \quad M=-\frac{1}{2} \operatorname{Im} \Omega, \quad N=H \Lambda^{2}-\frac{1}{2} \operatorname{Re} \Omega .
$$

Теперь задачу существования поверхности с заданными метрикой и средней кривизной можно формулировать так: рассматривается некоторая риманова поверхность $V$, компактная или открытая, с заданными на ней инвариантной относительно конформной замены координат метрической формой $d s^{2}=\Lambda^{2} d \zeta d \bar{\zeta}$ и числовой функцией $H$ и ищется изометрическое погружение $\mathbf{r}: V \rightarrow \mathbb{R}^{3}$, которое определяет в $\mathbb{R}^{3}$ поверхность $S=\mathbf{r}(V)$ со средней кривизной $H$. Локально (или, более общо́, для односвязного многообразия $V$, открытого или с краем) это равносильно тому, что ищется квадратичный дифференциал Хопфа $\Omega d \zeta^{2}$, в каждой карте удовлетворяющий уравнениям (3) и (4).

Коэффициент $\Omega$ дифференциала Хопфа обращается в нуль в омбилических точках поверхности, и только в них. Так как условие омбиличности задается равенством $H^{2}-K=0$, то все поверхности с заданной метрикой и заданной средней кривизной имеют на римановой поверхности $V$ совпадающие прообразы омбилических точек. Поэтому, говоря об омбилических точках поверхностей, мы будем иметь в виду не точки на самих поверхностях, а их прообразы на $V$.

Более тонкое свойство дается следующим утверждением.

ТеОрема 1. Пусть существуют две $C^{2}$-гладкие изометричные поверхности с общей средней кривизной. Тогда разность коэффициентов их дифберенциалов Хопфа обращается в нуль в омбилических и только в омбилических точках поверхностей. 
В литературе это утверждение хорошо известно, но обычно приводимое его доказательство нам представляется недостаточно обоснованным. Например, данное в [8] доказательство заведомо неверно в конечных классах гладкости, что подтверждается контрпримером ${ }^{1}$ к приведенному в [8; с. 20-21] рассуждению с равенством $\Omega_{1}+\Omega_{2}=g(z)=\left(z-z_{0}\right)^{J} g_{0}(z)$, справедливость которого при неявно предполагаемой $C^{\infty}$-гладкости поверхностей тоже требует более детального обоснования ${ }^{2}$. Как видно из формулировки, мы доказываем это утверждение для пары поверхностей Бонне класса $C^{2}$.

Сначала докажем следующую лемму.

ЛЕмма 1. В пределах одной карты разность коэффиииентов двух дифберенциалов Хопфа является голоморфной функцией.

ДокАзАтЕльство. Для поверхностей класса $C^{3}$ утверждение леммы очевидно, так как тогда коэффициенты дифференциалов Хопфа удовлетворяют системе $(3),(4)$ и разность $\Omega_{1}-\Omega_{2}$ ее двух решений имеет нулевую производную по $\bar{\zeta}$, т.е. удовлетворяет системе Коши-Римана. Для поверхностей класса $C^{2}$ дадим два доказательства. В первом доказательстве начнем с установления справедливости равенства

$$
\oint_{\partial D} \Omega d t=-2 \oint_{\partial D} H \Lambda^{2} d \bar{t}+2 \iint_{D} H \frac{\partial \Lambda^{2}}{\partial \zeta} d \zeta \wedge d \bar{\zeta}
$$

где $D$ - произвольная область со спрямляемой границей в области задания какой-либо карты поверхности. Действительно, для доказательства достаточно воспользоваться простыми равенствами

$$
\iint_{D} \frac{\partial w}{\partial \bar{\zeta}} d \zeta \wedge d \bar{\zeta}=-\oint_{\partial D} w(t) d t, \quad \iint_{D} \frac{\partial w}{\partial \zeta} d \zeta \wedge d \bar{\zeta}=\oint_{\partial D} w(t) d \bar{t}
$$

справедливыми для функций $w(z) \in C^{1}(D) \cap C(\bar{D})($ см. $[12 ;$ гл. $1, \S 4])$, и уравнением (4).

Пусть теперь рассматривается поверхность $S$ гладкости $C^{2}$. Ее можно аппроксимировать бесконечной последовательностью поверхностей $S_{n}$ гладкости $C^{3}$, сходящейся к ней в норме $C^{2}$ в некотором компакте, содержащем область $D$, и применить к поверхностям $S_{n}$ формулу вида (6). Тогда в пределе получим, что эта формула верна и для поверхностей гладкости $C^{2}$. После этого,

\footnotetext{
${ }^{1}$ Контрпример (в обозначениях работы [8]) дается функцией $g=i h \alpha=i\left(z-z_{0}\right)^{J} \times$ $h_{0}(z) \alpha(z) \in C^{J}$, где $h=\left(z-z_{0}\right)^{J} h_{0}(z), h_{0}\left(z_{0}\right) \neq 0,-$ голоморфная функция, $\alpha=$ $\left|z-z_{0}\right| \cos \left(2 \arg \left(z-z_{0}\right)\right) \notin C^{1}$ - действительная функция, но $g \in C^{J}$ (или даже $0<\alpha=$ $2+\cos \left(2 \arg \left(z-z_{0}\right)\right) \notin C$, но $\left.g \in C^{J-1}\right)$. Кроме того, даже в классе $C^{\infty}$ априори мы не можем утверждать, что порядок нуля функции $g$ не ниже порядка нуля функции $h$, и поэтому выполнение соотношений $\left|\frac{g}{h}\right|=i \alpha \in C$ или даже $\left|\frac{g}{h}\right|<$ const нельзя считать очевидно верным.

2 Это же рассуждение приведено и в $[9 ;$ с. 78,79$]$, однако в [9; с. 23] есть корректное доказательство, пригодное и для случая двух (а не только для непрерывного дифференцируемого семейства) поверхностей Бонне.
} 
предположив существование двух изометричных поверхностей с общим значением средней кривизны и соответственно с двумя функциями $\Omega_{1}$ и $\Omega_{2}$, удовлетворяющими соотношению (6), получим, что для любой жордановой спрямляемой кривой $\gamma$ имеет место равенство

$$
\oint_{\gamma}\left(\Omega_{1}(t)-\Omega_{2}(t)\right) d t=0
$$

что и является достаточным для голоморфности функции под знаком интегралом.

Дадим теперь второе доказательство леммы. Рассмотрим сначала поверхности класса $C^{3}$. Тогда решение системы $(4)$ имеет вид (см. [12; гл. 1])

$$
\Omega(\zeta)=T_{D} f+\Phi_{D}(\zeta)
$$

где интегральный оператор $T_{D}$ имеет вид

$$
T_{D} f=-\frac{1}{\pi} \iint_{D} \frac{f(z)}{z-\zeta} d \xi d \eta,
$$

a

$$
\Phi_{D}(\zeta)=\frac{1}{2 \pi i} \oint_{\partial D} \frac{\Omega(t)}{t-\zeta} d t
$$

- голоморфная функция. В книге [12] показано, что интегральные операторы

$$
T_{D} f \quad \text { и } \quad \Pi_{D} f=-\frac{1}{\pi} \iint_{D} \frac{f(z)}{(z-\zeta)^{2}} d \xi d \eta
$$

для функций класса $f \in C^{1}$ связаны соотношением

$$
T_{D}\left(\frac{\partial f}{\partial \zeta}\right)=\Pi_{D} f+\frac{1}{2 \pi i} \oint_{\partial D} \frac{f(t) d \bar{t}}{t-\zeta} .
$$

Учитывая, что $f=2 \Lambda^{2} H_{\zeta}=\left(2 \Lambda^{2} H\right)_{\zeta}-2 H\left(\Lambda^{2}\right)_{\zeta}$, для $\Omega(\zeta)$ получаем представление

$$
\Omega(\zeta)=\Pi_{D}\left(2 H \Lambda^{2}\right)-T_{D}\left(4 H \Lambda \Lambda_{\zeta}\right)+\Psi_{D}(\zeta)
$$

где

$$
\Psi_{D}(\zeta)=\Phi_{D}(\zeta)+\frac{1}{2 \pi i} \oint_{\partial D} \frac{2 H \Lambda^{2}}{t-\zeta} d \bar{t}
$$

- голоморфная функция.

Теперь для доказательства леммы достаточно аппроксимировать поверхность класса $C^{2}$ поверхностями класса $C^{3}$, сходящимися к ней в норме $C^{2}$, и, применив предельный переход, получить для $\Omega$ представление (8), в котором общие для всех решений системы (4) неголоморфные слагаемые зависят только от метрики и средней кривизны. Лемма доказана.

ЗАмЕчАНИЕ 1. Использованный сейчас метод может иметь более широкую область применения, так как он позволяет работать с системой ПетерсонаКодацци и в случае поверхностей гладкости $C^{2}$. А именно, формула (8) является для поверхностей класса $C^{2}$ аналогом уравнений Петерсона-Кодацци 
в том смысле, что вместе с уравнением Гаусса (3) выполнение равенства (8) необходимо для существования в односвязной области поверхности с заданными первой и второй квадратичными формами.

ЗАмЕчАниЕ 2. Вопрос о достаточности выполнения равенства (8) для существования $C^{2}$-гладкой поверхности пока остается открытым.

ДокАЗАТЕЛьство теоремы 1. Так как утверждение теоремы имеет локальный характер, можно считать, что все рассмотрения ведутся в некотором круге с центром в точке $z=0$. Пусть $\Omega_{1}(0)=\Omega_{2}(0)=a$, но пусть эта точка неомбилическая, т.е. $a \neq 0$. Положим

$$
\Omega_{1}(z)=a+\omega_{1}(z), \quad \Omega_{2}(z)=a+\omega_{2}(z), \quad \omega_{1}(0)=\omega_{2}(0)=0 .
$$

Так как разность $\Omega_{1}(z)-\Omega_{2}(z)$ в локальной карте является голоморфной функцией, то функция $\omega_{1}-\omega_{2}$ представима в виде $z \Phi(z)$, где $\Phi(z)$ - голоморфная функция. Тогда из равенства $\left(a+\omega_{1}\right)\left(\bar{a}+\bar{\omega}_{1}\right)=\left(a+\omega_{2}\right)\left(\bar{a}+\bar{\omega}_{2}\right)$ получаем уравнение

$$
\left(\bar{a}+\bar{\omega}_{1}\right) z \Phi(z)+\left(a+\omega_{2}\right) \bar{z} \bar{\Phi}=0 .
$$

Так как $\left|\Omega_{1}(z)\right|=\left|\Omega_{2}(z)\right|$, то можно положить

$$
\bar{a}+\bar{\omega}_{1}=R e^{i \varphi}, \quad a+\omega_{2}=R e^{i \psi}, \quad a=R_{0} e^{-i \varphi_{0}}=R_{0} e^{i \psi_{0}}, \quad R_{0}=|a| \neq 0 .
$$

Тогда приходим к уравнению

$$
\operatorname{Re}\left[\left(e^{i \varphi}+e^{-i \psi}\right) z \Phi\right]=0
$$

из которого получаем

$$
\cos \frac{\varphi+\psi}{2} \operatorname{Re}\left[e^{i \frac{\varphi-\psi}{2}} z \Phi\right]=0
$$

Допустим, есть последовательность точек с предельной точкой $z=0$, в которых $\cos \frac{\varphi+\psi}{2}=0$. Тогда в этих точках имеем $\omega_{1}+\omega_{2}=-2 a$, что в пределе приводит к противоречию с равенством $\omega_{1}(0)+\omega_{2}(0)=0$. Следовательно, такой последовательности нет, и мы можем найти окружность $|z|=r$, на которой уравнение (9) можно представить как так называемую краевую задачу Гильберта

$$
\operatorname{Re}\left[e^{i \frac{\varphi-\psi}{2}} t \Phi(t)\right]=0 .
$$

Так как $(\varphi-\psi) \rightarrow 2 \varphi_{0}$ при $z \rightarrow 0$, то индекс нашей задачи Гильберта есть

$$
\operatorname{Ind}\left(e^{i \frac{\psi-\varphi}{2}} \bar{t}\right)=-1<0
$$

а в таком случае однородная задача Гильберта имеет только нулевое решение (см. [13]). Значит, $a=0, \Omega(0)=\Omega_{2}(0)=0$, поэтому нуль разности $\Omega_{1}-\Omega_{2}$ совпадает с омбилической точкой. 
Теорема 1 имеет многочисленные следствия.

СледСтвиЕ 1. Поверхности, входящие в пару Бонне, имеют одинаковый класс гладкости.

СледСтвиЕ 2. Если существует пара Бонне поверхностей гладкости $C^{2}$, то омбилические точки соответствующих поверхностей расположены изолированно; в частности, если система (3), (4) имеет более одного решения, то нули этих решений изолированы и совпадают.

СледСтвиЕ 3. Если $C^{0}$-гладкий дифференциал Хопфа существует на всей римановой поверхности $V$, то он определяется единственным образом по его значениям в сколь угодно малой области $D \subset V$.

Действительно, пусть на $V$ есть два дифференциала Хопфа с коэффициентами $\Omega_{1}$ и $\Omega_{2}$, совпадающими в какой-либо области $D$ в общей параметризации. Тогда $\left(\Omega_{1}-\Omega_{2}\right) d z^{2}$ будет голоморфным дифференциалом с нулевым значением в $D$, поэтому он равен нулю на всем $V$, т.е. $\Omega_{1}=\Omega_{2}$ всюду.

СлЕДСтвиЕ 4. Если в классе $C^{3}$-гладких поверхностей система (3), (4) имеет в некоторой области $U \subset V$ ровно $n \leqslant 2$ решений, то на всей римановой поверхности $V$ она имеет не более $n$ решений. $B$ классе $C^{2}$-гладких поверхностей то же самое верно, если говорить о самих поверхностях, а не о решениях системы (т.е. если при данных на $V$ метрике и функиии $H$ средней кривизны некоторая область $U \subset V$ допускает ровно $n \leqslant 2$ соответствующих изометрических погружений гладкости $C^{2}$, то и все многообразия допускают не более $n$ погружений).

ЗАмЕчАНИЕ 3. Для правильного понимания этого утверждения нужно иметь в виду, что в принципе в неаналитическом классе возможна ситуация, когда существующая в $U$ поверхность может быть продолжима за пределы $U$ многими способами с сохранением даже $C^{\infty}$-гладкости. Из следствия 4 не вытекает, что система $(3),(4)$ ни в какой области не может иметь больше $n$ решений; из него следует лишь, что если в некоторой области $U_{1} \subset V$ система (3), (4) имеет $m>n$ решений, то какие-то $m-n$ решений не продолжимы в область $U$.

Случай наличия $n>2$ решений, как мы увидим позже, требует отдельного рассмотрения.

ЗАмЕчАние 4. Формула (6) допускает следующее обобщение:

$$
\oint_{\partial D} F(t) \Omega d t=-2 \oint_{\partial D} F H \Lambda^{2} d \bar{t}+2 \iint_{D} H \frac{\partial\left(F(\zeta) \Lambda^{2}\right)}{\partial \zeta} d \zeta \wedge d \bar{\zeta}
$$

где $F(\zeta)$ - любая голоморфная в $D$ функция. В этом обобщении, в частности, заключается справедливость формулы (6) при конформной замене системы координат. 
Теорема 1 уже накладывает ограничения на возможность найти для данной поверхности ее пару Бонне: для поверхностей, составляющих пару Бонне, их дифференциалы Хопфа не могут быть равными ни в одной неомбилической точке (конечно, при вычислении их в общей карте). По-другому, в силу непрерывности и необращения в нуль дифференциалов Хопфа в неомбилической точке можно сказать, что эти дифференциалы не эквивалентны при подходе к неомбилической точке. В омбилической точке эти два дифференциала Хопфа, будучи равными нулю, равны между собой, но при подходе к омбилической точке они по-прежнему не будут эквивалентными. Это свойство мы сформулируем как следующее

УтвеРЖДЕНИЕ 1. На $C^{\infty}$-гладжих изометричных поверхностях с общей средней кривизной при подходе $\kappa$ омбилической точке $p_{0} \in V$ коэфбициенты $\Omega_{1}$ и $\Omega_{2}$ дифференциалов Хопфа в общей системе локальных координат не могут быть эквивалентными и их тейлоровы разложения начинаются с многочленов одной и той же степени $n$, где $n$ - кратность нуля голоморфной функиии $\Omega_{1}-\Omega_{2}$ в омбилической точке $p_{0}$.

ДокАЗАТЕЛЬСтво. Пусть омбилической точке соответствует координата $\zeta=0$. В силу голоморфности функции $h(\zeta)=\Omega_{1}-\Omega_{2}$ имеем представление $h=\zeta^{n} h_{0}(\zeta), n \geqslant 1, h_{0}(0)=c \neq 0$. Тогда не может быть, чтобы тейлоровы разложения обеих функций $\Omega_{1}$ и $\Omega_{2}$ начинались с некоторой степени $m>n$. Пусть хотя бы для одной из функций $\Omega_{j}$, скажем, для $\Omega_{1}$, в ее тейлоровом разложении первый раз встретился ненулевой однородный многочлен $T_{m}(\xi, \eta)$ некоторой степени $m, 1 \leqslant m<n$, где $\xi+i \eta=\zeta$. Тогда в силу представления $\Omega_{1}-\Omega_{2} \sim c \zeta^{n}, c \neq 0$, тейлорово разложение для $\Omega_{2}(\zeta)$ тоже начнется с $T_{m}(\xi, \eta)$. И так как $m<n$, то в обоих разложениях будут также многочлены степени $n$, соответственно $T_{n}^{(1)}(\xi, \eta)$ и $T_{n}^{(2)}(\xi, \eta)$, из которых хотя бы один не является нулевым, и $T_{n}^{(1)}(\xi, \eta)-T_{n}^{(2)}(\xi, \eta) \not \equiv 0$, а для всех $k, m \leqslant k \leqslant n-1$, имеем $T_{k}^{(1)}(\xi, \eta)-T_{k}^{(2)}(\xi, \eta) \equiv 0$. Получаем представления

$$
\Omega_{1}=Q_{0}(\xi, \eta)+Q_{1}(\xi, \eta), \quad \Omega_{2}=Q_{0}(\xi, \eta)+Q_{2}(\xi, \eta),
$$

где

$$
\begin{gathered}
Q_{0}=T_{m}(\xi, \eta)+\cdots+T_{n-1}(\xi, \eta) \\
Q_{1}=T_{n}^{(1)}(\xi, \eta)+\cdots, \quad Q_{2}=T_{n}^{(2)}(\xi, \eta)+\cdots
\end{gathered}
$$

Из условия $\left|\Omega_{1}(\zeta)\right|=\left|\Omega_{2}(\zeta)\right|$ получаем равенство

$$
\begin{aligned}
\left(T_{m}(\xi, \eta)\right. & \left.+\cdots+T_{n-1}(\xi, \eta)\right) \bar{h}(\zeta)+\left(\bar{T}_{m}(\xi, \eta)+\cdots+\bar{T}_{n-1}(\xi, \eta)\right) h(\zeta) \\
& +Q_{1}(\xi, \eta) \bar{Q}_{1}(\xi, \eta)-Q_{2}(\xi, \eta) \bar{Q}_{2}(\xi, \eta)=0
\end{aligned}
$$

из которого с учетом представления $h(\zeta)=\zeta^{n} h_{0}(\zeta), h_{0}(0) \neq 0$, получаем

$$
T_{m}(\xi, \eta) \bar{\zeta}^{n} \bar{h}_{0}(0)+\bar{T}_{m}(\xi, \eta) \zeta^{n} h_{0}(0)=0 .
$$


А это уравнение при $m<n$ выполнимо, только если $T_{m}(\xi, \eta) \equiv 0$. Следовательно, тейлоровы разложения функций $\Omega_{1}$ и $\Omega_{2}$ должны начинаться с ненулевых и не совпадающих многочленов одной и той же степени $n$, где $n-$ порядок нуля голоморфной функции $h=\Omega_{1}-\Omega_{2}$, что и утверждалось.

На самом деле для тейлоровых разложений функций $\Omega_{j}, j=1,2$, в окрестности любой точки можно получить представление, которое в окрестности омбилической точки имеет более информативное содержание, чем утверждение 1 , а именно верно следующее

УтвеРЖДЕНИЕ 2. Тейлоровы разложения функиий $\Omega_{j}, j=1,2$, для пар Бонне гладкости $C^{\infty}$ в окрестности любой точки имеют вид

$\Omega_{1}=\alpha_{1} \zeta^{n}+b_{1} \zeta^{n+1}+c \bar{\zeta} \zeta^{n}+o\left(|\zeta|^{n+1}\right), \quad \Omega_{2}=\alpha_{2} \zeta^{n}+b_{2} \zeta^{n+1}+c \bar{\zeta} \zeta^{n}+o\left(|\zeta|^{n+1}\right)$,

где $n \geqslant 0$ - порядок нуля голоморфной функции $\varphi=\Omega_{1}-\Omega_{2}$ в рассматриваемой точке, $\alpha_{1} \neq \alpha_{2},\left|\alpha_{1}\right|=\left|\alpha_{2}\right|$, а разность остатков является голоморфной функцией с порядком нуля не ниже $n+2$ (как обычно, считаем, что рассматриваемой точке р соответствует параметр $\left.\zeta_{0}=0\right)$.

ДокАЗАТЕЛЬСтво. Для случая неомбилической точки $n=0$ и утверждение очевидно. Пусть точка омбилическая. В силу утверждения 1 для $j=1,2$ имеем представления

$$
\Omega_{j}=a_{j} \zeta^{n}+\sum_{k=1}^{n} b_{j k} \bar{\zeta}^{k} \zeta^{n-k}+b_{j} \zeta^{n+1}+\sum_{k=1}^{n+1} c_{j k} \bar{\zeta}^{k} \zeta^{n+1-k}+o\left(|\zeta|^{n+1}\right), \quad|\zeta| \rightarrow 0
$$

Так как разность $\Omega_{1}-\Omega_{2}$ голоморфна и имеет в точке $p$ нуль порядка $n$, имеем соотношения

$$
a_{1} \neq a_{2}, \quad b_{1 k}=b_{2 k}, \quad 1 \leqslant k \leqslant n, \quad c_{1 k}=c_{2 k}, \quad 1 \leqslant k \leqslant n+1 .
$$

Далее, учитывая равенство $\left|\Omega_{1}\right|=\left|\Omega_{2}\right|$ и приравнивая в нем коэффициенты при одинаковых степенях малости порядка $2 n$ относительно $|\zeta| \rightarrow 0$, получаем

$$
\left|a_{1}\right|=\left|a_{2}\right|, \quad \operatorname{Re}\left(\sum_{k=1}^{n}\left(a_{1}-a_{2}\right) \bar{b}_{1 k} e^{2 k i \varphi}\right)=0,
$$

что дает равенства $b_{1 k}=b_{2 k}=0,1 \leqslant k \leqslant n$. Аналогичным образом, приравнивая в равенстве коэффициенты при степенях малости порядка $2 n+1$, получаем

$$
\left(a_{1}-a_{2}\right) \bar{c}_{11}=b_{2} \bar{a}_{2}-b_{1} \bar{a}_{1}, \quad c_{1 k}=0, \quad 2 \leqslant k \leqslant n+1,
$$

что и требовалось показать. В общем случае $c=c_{11} \neq 0$.

Из этого утверждения получаем два следствия.

СледСтвиЕ 5. На парах Бонне класса гладкости $C^{\infty}$ порядок обращения в нуль функиий $\Omega_{j}, j=1,2$, в омбилической точке совпадает с порядком нуля голоморфной функиии $\Omega_{1}-\Omega_{2}$ в этой точке. В частности, при стремлении $\kappa$ омбилической точке с координатой $\zeta=0$ имеем $g \sim C|\zeta|^{2 n}, C>0$, где $n \geqslant 1$ - порядок нуля голоморфной функции $\Omega_{1}-\Omega_{2}$ в точке $\zeta=0$. 


\section{Особенно важным является}

СЛЕДСТВиЕ 6. При стремлении $к$ любой точке $C^{\infty}$-гладкой поверхности из пары Бонне отношение $\frac{\Omega_{1}}{\Omega_{2}}$ стремится $\kappa$ определенному пределу, отличному от 1 , тем самым, отношение $\frac{\Omega_{1}}{\Omega_{2}}$ продолжимо по непрерывности как непрерывная функиия на всю поверхность.

Доказательство. Действительно, числовая функция $\theta(\zeta)$, определяемая равенством $\Omega_{2}=\Omega_{1} e^{i \theta(\zeta)}$, существует на всей поверхности, непрерывна всюду и однозначна, так как вне омбилических точек $e^{i \theta} \neq 1$; значит, если в некоторой точке выбрано значение $\theta \in(0,2 \pi)$, то $0<\theta<2 \pi$ во всех неомбилических точ$\mathrm{kax}^{3}$, поэтому при обходе вокруг любой омбилической точки функция $\theta$ должна возвратиться к исходному значению, а в омбилической точке она в силу утверждения 2 имеет предел, отличный от нуля и $2 \pi$.

ЗАмЕчАниЕ 5. В случае конечных классов гладкости поверхностей в паре Бонне утверждение следствия 6 остается верным, если заранее предположить, что порядок гладкости функций $\Omega_{j}$ не ниже, чем порядок $n$ нуля голоморфной функции $h=\Omega_{2}-\Omega_{1}$, так как в этом случае для доказательства достаточно иметь представление (10) только с главными членами порядка $n$. В частности, если на поверхности нет омбилических точек (случай тора) или все нули этой функции $h$ простые, то следствие 6 верно для поверхностей гладкости $C^{3}$. Без предположения нужной гладкости вопрос о поведении отношения $\frac{\Omega_{1}}{\Omega_{2}}$ при подходе к омбилической точке остается открытым ${ }^{4}$. Поэтому при малых гладкостях поверхностей из пары Бонне о функции $\theta$ можно сказать лишь, что она существует, однозначна и ограничена на поверхности всюду вне омбилических точек, а возможность ее доопределения в омбилических точках требует специального рассмотрения.

\section{§ 3. Некоторые необходимые/достаточные условия существования пар Бонне}

Приведем несколько полезных необходимых/достаточных условий существования пары Бонне для заданной поверхности.

УтвеРЖДЕНИЕ 3. Пусть дана некоторая поверхность $S_{1} \in C^{3}$. Тогда для того, чтобъ $S_{1}$ имела парную по Бонне поверхность $S_{2}$, необходимо, а в односвлзном случае и достаточно, чтобы на $S_{1}$ существовали голоморфный квадратичный дифференциал $\varphi d \zeta^{2}$ и вещественная функиия $\theta$ такие, что коэффициент $\Omega_{1}$ дифференциала Хопфа поверхности $S_{1}$ имеет представление

$$
\Omega_{1}=-\frac{1}{2}\left(1+i \operatorname{ctg} \frac{\theta}{2}\right) \varphi
$$

\footnotetext{
${ }^{3}$ Вместо этого конкретного условия можно предполагать выполнение более общего условия $\sin \frac{\theta}{2} \neq 0$.

${ }^{4}$ Открытым остается и вопрос о представлении суммы $G=\Omega_{1}+\Omega_{2}$ в виде $G=\left(\zeta-\zeta_{0}\right) G_{0}(\zeta)$ с некоторой гладкой функцией $G_{0}$ около точки, в которой эта сумма обращается в нуль.
} 
причем существующии тогда коэффичиент $\Omega_{2}$ дифференциала Хопфа поверхности $S_{2}$ будет иметь представление

$$
\Omega_{2}=\frac{1}{2}\left(1-i \operatorname{ctg} \frac{\theta}{2}\right) \varphi .
$$

ДокАзАтЕльство. Действительно, при существовании пары Бонне вводим голоморфную функцию $\varphi=\Omega_{2}-\Omega_{1}$ и рассмотренную выше (в $\S 2$ ) функцию $\theta$ с $\Omega_{2}=\Omega_{1} e^{i \theta}$. Тогда представления (11) и (12) получаются простыми преобразованиями.

Обратно, пусть поверхность $S_{1}$ имеет метрический коэффициент $\Lambda$ и среднюю кривизну $H$, и пусть $\Omega_{1}$ имеет представление (11). Коэффициенты второй квадратичной формы поверхности $S_{1}$ определены соответствующей формулой (5). Возьмем ту же самую метрическую форму, а коэффициенты второй квадратичной формы возьмем по той же самой формуле (5) с использованием $\Omega_{2}$ из (12). Легко проверить, что $\Omega_{1}$ и $\Omega_{2}$ удовлетворяют одной и той же системе (3) и тогда в односвязном случае обе поверхности однозначно определяются двумя своими квадратичными формами, составляя пару Бонне.

Для дальнейшего нам понадобится следующая формула, связывающая коэффициенты $\Omega, \varphi$ и функцию $\theta$ :

$$
g=\frac{\varphi \bar{\varphi}}{4 \sin ^{2} \frac{\theta}{2}}, \quad g=\Omega \bar{\Omega} .
$$

Заметим, что необходимость условий очевидно верна и в классе гладкости $C^{2}, \mathrm{a}$ доказательство достаточности в этом классе гладкости потребует, по-видимому, довольно серьезных усилий (см. замечания 1 и 2).

Дадим еще один критерий существования пары Бонне для данной поверхности.

УТВеРЖДЕНИЕ 4. Для того чтобъ данная поверхность $S_{1}$ имела парную ей поверхность Бонне, необходимо, а в односвязном случае и достаточно, чтобы на $S_{1}$ существовал голоморфный квадратичный дифференциал $\varphi d \zeta^{2}$, который связан с дифференциалом Хопфа поверхности $S_{1}$ соотношением

$$
\bar{\varphi} \Omega_{1}+\varphi \bar{\Omega}_{1}=-\varphi \bar{\varphi}
$$

причем существующий тогда коэффищиент $\Omega_{2}$ равен $\Omega_{1}+\varphi$ и для него верно равенство

$$
\bar{\varphi} \Omega_{2}+\varphi \bar{\Omega}_{2}=\varphi \bar{\varphi}
$$

ДокАЗАтельство. Необходимость доказывается тривиально, докажем достаточность. Пусть для поверхности $S_{1}$ выполняются условия утверждения. Тогда, во-первых, очевидно, что в омбилических точках поверхности имеем нуль функции $\varphi$, во-вторых, менее очевидно, но все же легко доказать от противного, что в неомбилических точках поверхности функция $\varphi$ не может обращаться в нуль. Положим $\Omega_{2}=\Omega_{1}+\varphi$; в силу условия (14) имеем $\left|\Omega_{2}\right|=\left|\Omega_{1}\right|$ и уравнения (3) выполнены. 
В заключение этого параграфа выясним геометрический смысл функции $\theta$. Так как вне омбилических точек голоморфная функция $\varphi$ не равна нулю, то там за счет выбора новых координат можно добиться, чтобы в новых координатах стало $\varphi \equiv 1$. Тогда для коэффициентов вторых квадратичных форм поверхностей из пары Бонне соответственно получим

$$
\begin{array}{lll}
L_{1}=H \Lambda^{2}-\frac{1}{4}, & M_{1}=\frac{1}{4} \operatorname{ctg} \frac{\theta}{2}, & N_{1}=H \Lambda^{2}+\frac{1}{4}, \\
L_{2}=H \Lambda^{2}+\frac{1}{4}, & M_{2}=\frac{1}{4} \operatorname{ctg} \frac{\theta}{2}, & N_{1}=H \Lambda^{2}-\frac{1}{4} .
\end{array}
$$

Геометрический смысл функции $\theta$ раскрывается следующим утверждением: если на многообразии $V$, два изометрических погружения в $\mathbb{R}^{3}$ которого дают две поверхности $S_{1}$ и $S_{2}$ из пары Бонне, рассмотреть главные направления, соответствующие этим поверхностям и одинаковым главным кривизнам, то угол между ними будет равен $\frac{1}{2} \theta$.

\section{§ 4. Дифференциал Хопфа как функция метрики и средней кривизны}

Теперь покажем, что для поверхностей гладкости $C^{5}$ в общем случае $\Omega$ определяется в явном виде как некоторое алгебраическое выражение от функций $f, g$ и их производных, т.е. в конечном счете как элементарная функция от средней кривизны, метрического коэффициента и их производных.

Для этого продифференцируем уравнение (3) по $\partial \bar{\zeta}$ и с учетом (4) получим

$$
f \bar{\Omega}+\Omega \frac{\overline{\partial \Omega}}{\partial \zeta}=\frac{\partial g}{\partial \bar{\zeta}}
$$

Умножим полученное выражение на $\bar{\Omega}$, заменим произведение $\Omega \bar{\Omega}$ на $g$ и после дифференцирования по $\partial \zeta$ с последующим умножением на $\Omega$ и учетом уравнений $(3),(4)$ и (16) придем к соотношению

$$
\left(g \frac{\partial f}{\partial \zeta}-f \frac{\partial g}{\partial \zeta}\right) \bar{\Omega}+\left(g \frac{\overline{\partial f}}{\partial \zeta}-\bar{f} \frac{\partial g}{\partial \bar{\zeta}}\right) \Omega=g \frac{\partial^{2} g}{\partial \zeta \partial \bar{\zeta}}-\frac{\partial g}{\partial \zeta} \frac{\partial g}{\partial \bar{\zeta}}-2 g f \bar{f} .
$$

Положим ${ }^{5}$

$$
A=g \frac{\partial f}{\partial \zeta}-f \frac{\partial g}{\partial \zeta}, \quad B=g \frac{\partial^{2} g}{\partial \zeta \partial \bar{\zeta}}-\frac{\partial g}{\partial \zeta} \frac{\partial g}{\partial \bar{\zeta}}-2 g f \bar{f}=g^{2} \frac{\partial^{2} \ln g}{\partial \zeta \partial \bar{\zeta}}-2 g f \bar{f}
$$

и запишем уравнение (17) в виде

$$
A \bar{\Omega}+\bar{A} \Omega=B .
$$

\footnotetext{
${ }^{5}$ Нетрудно проверить, что введенные величины $A$ и $B$ образуют дифференциалы $A(\zeta) d \zeta^{5} d \bar{\zeta}^{3}$ и $B(\zeta) d \zeta^{5} d \bar{\zeta}^{5}$ порядков $(5,3)$ и $(5,5)$ с соответствующим изменением коэффициентов при конформной замене координат; в частности, из этого следует, что знак коэффициента $B$ и неравенство/равенство нулю коэффициента $A$ от выбора системы конформных координат не зависят.
} 
Из этого уравнения можно, во-первых, умножением (19) на $\Omega$ получить квадратное уравнение

$$
\bar{A} \Omega^{2}-B \Omega+g A=0,
$$

из которого и найдутся два значения $\Omega$, во-вторых, можно прийти к линейному уравнению. Для этого продифференцируем (19) по $\partial \bar{\zeta}$, заменим в результате $\frac{\partial \Omega}{\partial \bar{\zeta}}$ на $f$, затем умножим на $\Omega$ и подставим вместо произведения $\Omega \frac{\overline{\partial \Omega}}{\partial \zeta}$ его значение из (16). Получим уравнение

$$
A g_{\bar{\zeta}}+g A_{\bar{\zeta}}+\left(f \bar{A}-B_{\bar{\zeta}}\right) \Omega-f A \bar{\Omega}+\bar{A}_{\zeta} \Omega^{2}=0 .
$$

Исключим $\Omega^{2}$ из этого уравнения и из $(20)$, а затем из полученного выражения и из $(19)$, исключив $\bar{\Omega}$, получим окончательный результат

$$
C \Omega=D
$$

где

$$
C=2 f \bar{A}^{2}+B \overline{A_{\zeta}}-\bar{A} B_{\bar{\zeta}}, \quad D=g A \overline{A_{\zeta}}-g \bar{A} A_{\bar{\zeta}}-\bar{A} A g_{\bar{\zeta}}+f B \bar{A}
$$

Следовательно, если $C \neq 0$, то $\Omega$ находится единственным и явным образом через $f, g$ и их производные по формуле $\Omega=\frac{D}{C}$. Однако нужно еще убедиться, что $\frac{D \bar{D}}{C \bar{C}}=g$ и $\frac{\partial}{\partial \bar{\zeta}}\left(\frac{D}{C}\right)=f$. Посмотрим, когда это верно, причем для нужной проверки нам не придется даже вычислять требуемую производную (т.е. мы сможем обойтись без формального предположения $C^{6}$-гладкости рассматриваемой поверхности).

На самом деле, если $C \neq 0$, то и $A \neq 0$ (действительно, если предположить противное, $A=0$, то из (19) имеем $B=0$, поэтому $C=0)$. А при $A \neq 0$ уравнение (20) разрешимо и имеет два решения:

$$
\Omega=\frac{B \pm \sqrt{B^{2}-4 g A \bar{A}}}{2 \bar{A}} .
$$

Убедимся, что выражение под корнем не может быть положительным. Допустим обратное: $B^{2}-4 g A \bar{A}>0$. Тогда в числителе дроби для $\Omega$ имеем действительную функцию и, подставив найденное решение в уравнение (19), получим

$$
A \frac{B \pm \sqrt{B^{2}-4 g A \bar{A}}}{2 A}+\bar{A} \frac{B \pm \sqrt{B^{2}-4 g A \bar{A}}}{2 \bar{A}}=B \pm \sqrt{B^{2}-4 g A \bar{A}}=B
$$

что невозможно. Значит, $B^{2}-4 g A \bar{A} \leqslant 0$, поэтому

$$
\Omega=\frac{B \pm i \sqrt{4 g A \bar{A}-B^{2}}}{2 \bar{A}} .
$$

Прямым вычислением можно убедиться, что для обоих решений выполнено необходимое равенство $|\Omega|^{2}=g$. 
Далее, при $C \neq 0$ невозможно, чтобы было $4 g A \bar{A}-B^{2}=0$. Действительно, предположим противное: пусть в некоторой точке $4 g A \bar{A}-B^{2}=0$. Представим (23) и $C$ из $(22)$ как

$$
\Omega-\frac{B}{2 \bar{A}}= \pm i \frac{\sqrt{4 g A \bar{A}-B^{2}}}{2 A \bar{A}} A, \quad C=2 \bar{A}^{2}\left(f-\left(\frac{B}{2 \bar{A}}\right)_{\bar{\zeta}}\right) .
$$

Функция

$$
F=\frac{\sqrt{4 g A \bar{A}-B^{2}}}{2 A \bar{A}} A=\frac{\sqrt{4 g A \bar{A}-B^{2}}}{2 \bar{A}}
$$

принадлежит классу $C^{1}$, потому что она равна $\mp i\left(\Omega-\frac{B}{2 \bar{A}}\right) \in C^{1}$. Следовательно, функция

$$
\frac{\sqrt{4 g A \bar{A}-B^{2}}}{2 A \bar{A}}=\frac{F}{A}
$$

тоже имеет производные первого порядка. А так как она неотрицательная и по предположению равна нулю в рассматриваемой точке, то в этой точке ее производные равны нулю. Значит,

$$
\left(\Omega-\left(\frac{B}{2 \bar{A}}\right)\right)_{\bar{\zeta}}=f-\left(\frac{B}{2 \bar{A}}\right)_{\bar{\zeta}}=0,
$$

что дает равенство $C=0$ в противоречии с условием $C \neq 0$.

Итак, при $C \neq 0$ имеем $A \neq 0$ и $4 g A \bar{A}-B^{2}>0$. Но тогда одна из двух существующих по формуле (23) функций $\Omega$ должна удовлетворять уравнению (4). Это приводит к необходимости выполнения равенства

$$
C \sqrt{4 g A \bar{A}-B^{2}}=i \varepsilon\left[\overline{A_{\zeta}}\left(4 g A \bar{A}-B^{2}\right)-\bar{A}\left(2(g A \bar{A})_{\bar{\zeta}}-B B_{\bar{\zeta}}\right)\right], \quad \varepsilon=\mp 1,
$$

правую часть которого можно представить в виде $i \varepsilon(2 D \bar{A}-B C)$. Следовательно, условие (24) сводится к выполнению соотношения $C \Omega=D$, в котором $\Omega$ является одним из корней (23). Это значит, что если $\Omega=\frac{D}{C}$ удовлетворяет необходимому условию быть одним из решений уравнения (20), т.е.

$$
\frac{B+i \varepsilon \sqrt{4 g A \bar{A}-B^{2}}}{2 \bar{A}}=\frac{D}{C}
$$

с некоторым $\varepsilon= \pm 1$, то для этого решения выполнено равенство $\Omega_{\bar{\zeta}}=f$, что проверяется довольно длинным, но прямым вычислением.

Таким образом, с учетом следствия 1 доказана следующая

Теорема 2. Если для метрики (1) и средней кривизны Н поверхности $S \in C^{5}$ выполнено условие $C \neq 0$, где $C$ определено равенством (22), то поверхность $S$ не имеет свей парь Бонне даже в классе поверхностей гладкости $C^{2}$, а для введенных коэфбициентов $f, g, A, B, C$ и $D$ выполнены неравенства $g>0, A \neq 0,4 g A \bar{A}-B^{2}>0$ и одно из уравнений (25) с $\varepsilon=1$ или $\varepsilon=-1$. Обратно, если на открытой односвязной римановой поверхности $V$ заданы 
метрическая форма (1) с $\Lambda \in C^{n}, 5 \leqslant n \leqslant \infty, u$ функиия $H \in C^{n-2}$, для которых построенные по ним выражсения $f, g, A, B, C, D$ удовлетворяют неравенствам $g>0, A \neq 0, C \neq 0,4 g A \bar{A}-B^{2}>0$ и одному из уравнений (25), то в $\mathbb{R}^{3}$ существует единственная поверхность, имеющая метрику (1) и среднюю кривизну $H$, причем она будет класса гладкости $C^{n}$ (и аналитическая, если $\Lambda$ и нвляются аналитическими).

ЗАмЕчАниЕ 6 . В силу следствия 4 для несуществования пары Бонне для поверхности $S \in C^{5}$ достаточно, чтобы хотя бы в одной некоторой точке $p \in S$ было $C(p) \neq 0$.

ЗАмечАниЕ 7. Из изложенного выше получаем, что так как поверхность принадлежит классу $C^{5}$, то одна из правых частей выражений для $\Omega$ из $(23)$, формально имеющая гладкость $C^{1}$, и решение $\Omega=\frac{D}{C}$ уравнения $(21)$, формально являющееся только непрерывным, на самом деле имеют гладкость класса $C^{3}$ (еще один пример "парадокса дифференцируемости” в теории поверхностей, о котором писали Хартман и Уитнер в [14]).

Итак, случай $C \neq 0$ разобран полностью: он дает неравенства $A \neq 0$, $4 g A \bar{A}-B^{2}>0$ и при нем возможно существование только одной поверхности с заданными метрикой и средней кривизной. Важно заметить, что для единственности поверхности достаточно иметь неравенство $C \neq 0$ не всюду на многообразии $V$, а хотя бы в некоторой одной точке $p \in V$.

Отметим, что комплексное дифференциальное уравнение (25) в равной степени связывает метрику и среднюю кривизну (оно третьего порядка относительно $H$ и пятого порядка относительно $\Lambda$ ), и поэтому можно сформулировать следующие вопросы: для каких функций $H$ (соответственно метрического коэффициента $\Lambda$ ), заданных на некотором многообразии, можно подобрать метрический коэффициент $\Lambda$ (соответственно функцию $H$ ) так, чтобы они в паре определяли нужную поверхность? Поиск функции $H$ по заданному метрическому коэффициенту $\Lambda$ равносилен решению задачи об изометрическом погружении в $\mathbb{R}^{3}$ данной метрики, а задачу поиска $\Lambda$ по заданной функции $H$ можно сформулировать так: всякая ли функция $H$ является средней кривизной некоторой поверхности? Нам не известно, рассматривалась ли в литературе такая задача.

ЗАмЕчАниЕ 8. Комплексное уравнение (25) дает для двух действительных функций систему из двух уравнений. Предположим, что она при любой данной функции $\Lambda$ с $\Lambda(0)=1$ имеет некоторое решение $H$. Тогда это означало бы, что любая метрика локально изометрически погружается в $\mathbb{R}^{3}$ (в соответствующих предположениях гладкости) и, тем самым, было бы дано положительное решение проблемы о локальной погружаемости любой метрики. С другой стороны, представляется маловероятным, чтобы система для пары функций $(H, \Lambda)$ имела решение с любым выбором второй компоненты, поэтому, скорее всего, следует ожидать, что существуют локально непогружаемые метрики класса $C^{\infty}$, или, что то же самое, что отображение $P: H \rightarrow \Lambda$, сопоставляющее каждой функции $H$ по уравнению (25) его решения $\Lambda$, не является отображением "на" 
множество метрик (т.е. оно не сюръективно). Более того, по-видимому, можно высказать такую гипотезу: в классе метрик $d s^{2}=\Lambda^{2}\left(d u^{2}+d v^{2}\right) \in C^{\infty}$ со знакопеременной кривизной $K$ и с условием $\operatorname{grad} K(0,0)=0$ "почти все" метрики даже локально не допускают изометрического погружения в $\mathbb{R}^{3}$. Здесь слова "почти все" можно понимать как условие выполнения некоторого алгебраического или дифференциального равенства для погружаемых метрик (в то же время это условие необходимо должно выполняться для всех аналитических метрик, что является одним из интригующих свойств этого гипотетического равенства).

ЗАмЕчАниЕ 9. Слишком "суровые” условия связи между метрикой и средней кривизной искомой поверхности естественно приводят к задаче нахождения поверхности только по ее гауссовой и средней кривизнам, или, что равносильно, по заданному распределению ее главных кривизн.

Завершим этот параграф некоторой подготовкой перехода к рассмотрению случаев $C=0$. Для поверхностей с $C=0$ полезно иметь в виду следующие утверждения.

УтВеРЖДЕНИЕ 5. Если для данной поверхности $S$ в некоторой точке $p$ имеем $C(p)=0$, то в этой точке и $D(p)=0$.

ДоказАТЕЛЬСтво. Действительно, если $D(p) \neq 0$, то для $D$ из $(22)$ видим, что и $A(p) \neq 0$. Значит, функцию $\Omega$ для поверхности $S$ можно выразить в виде (23). А тогда равенство

$$
\frac{\partial \Omega}{\partial \bar{\zeta}}=f
$$

сводится к равенству (24), записанному в виде

$$
C \sqrt{4 g A \bar{A}-B^{2}}=i \varepsilon(2 D \bar{A}-B C),
$$

которое при $C(p)=0, D(p) \neq 0$ не выполнимо.

УтВеРЖДЕНИЕ 6. В омбилических точках $A=B=C=0$.

ДокАЗАТЕЛЬСтво. Действительно, в окрестности омбилической точки $p$ имеем $g \geqslant 0$ и в то же время $g(p)=0$. Значит,

$$
\frac{\partial g}{\partial \zeta}(p)=0 \quad \Longrightarrow \quad A(p)=0 \quad \Longrightarrow \quad B(p)=0 \quad \Longrightarrow \quad C(p)=0 .
$$

\section{§ 5. Один признак несуществования пары Бонне}

Есть еще один случай, когда существующая при данных метрике и средней кривизне поверхность единственна. А именно, пусть $C \equiv 0$, но $A \neq 0$ и $\Delta=: 4 g A \bar{A}-B^{2} \equiv 0$. Тогда уравнение (20) имеет единственное решение $\Omega=\frac{B}{2 A}$, для которого равенство $\frac{\partial \Omega}{\partial \bar{\zeta}}=f$ выполняется как раз в силу наличия условия $C=0$. Этот класс поверхностей является в некотором смысле выделенным случаем локальной единственности поверхности с данными метрикой 
и средней кривизной. Покажем, что класс таких поверхностей не пуст. Начнем с установления следующего их свойства.

УТВЕРЖДЕНИЕ 7. Если для поверхности выполнены условия

$$
C \equiv 0, \quad A \neq 0, \quad \Delta=4 g A \bar{A}-B^{2} \equiv 0,
$$

то $\arg \Omega$ является гармонической функцией. Обратно, если $\arg \Omega$ является гармонической функиией, то $4 g A \bar{A}-B^{2} \equiv 0$.

ДокАзАТЕльство. Так как $A \neq 0$ (значит, по доказанному выше, $g \neq 0$ ), то можно положить

$$
A=|A| e^{i \theta}
$$

где $\theta=\arg A$. Далее, $B \neq 0$, иначе было бы $A=0$. Пусть $\operatorname{sgn} B=\varepsilon= \pm 1$. Тогда из условия $4 g A \bar{A}=B^{2}$ и из (23) имеем

$$
\Omega=\frac{B}{2 \bar{A}}=\varepsilon \sqrt{g} e^{i \theta}, \quad \theta=\arg (\varepsilon \Omega) .
$$

Кроме того, из равенства

$$
C=2 \bar{A}^{2}\left(f-\left(\frac{B}{2 \bar{A}}\right)_{\bar{\zeta}}\right)=0
$$

получаем, что $\Omega$ из (27) удовлетворяет уравнению (4). Дифференцируя (27) по $\frac{\partial}{\partial \bar{\zeta}}$, получим

$$
\theta_{\bar{\zeta}}=i \frac{g_{\bar{\zeta}}}{2 g}-i \varepsilon \frac{f e^{-i \theta}}{\sqrt{g}} .
$$

Дифференцируя полученное уравнение по $\frac{\partial}{\partial \zeta}$ и учитывая определения $A$ и $B$ из (18) и равенство (26), приходим к выражению

$$
-\theta_{\zeta \bar{\zeta}}=i \varepsilon \frac{|A|}{g \sqrt{g}}-i \frac{B}{2 g^{2}}=0,
$$

что и доказывает наше прямое утверждение.

Докажем обратное утверждение. Пусть $\Omega=\sqrt{g} e^{i \theta} \neq 0$, где $\theta$ - гармоническая функция, т.е. $\theta_{\zeta \bar{\zeta}}=0$. Имеем

$$
\frac{\partial \Omega}{\partial \bar{\zeta}}=\frac{g_{\bar{\zeta}}}{2 \sqrt{g}} e^{i \theta}+i \sqrt{g} e^{i \theta} \theta_{\bar{\zeta}}=f .
$$

Дифференцируя это равенство по $\frac{\partial}{\partial \zeta}$, после простых преобразований находим $B e^{i \theta}=2 \bar{A} \sqrt{g}$, что и дает нужное равенство $\Delta=4 g A \bar{A}-B^{2} \equiv 0$. Утверждение доказано.

Продолжим исследование поверхностей с условием $\Delta=0$. Из равенства $2 \Omega \bar{A}=B$ с условием $\operatorname{Im} B=0, B \neq 0$, следует, что нам известна гармоническая функция

$$
\arg \Omega=\arg A+\frac{1-\operatorname{sgn} B}{2} \pi
$$


Построим по этой гармонической функции $\arg \Omega$ голоморфную функцию $h(\zeta) \neq 0$ такую, что $\ln h(\zeta)=\ln |h|+i \arg \Omega$, затем сделаем конформную замену переменной $z=z(\zeta)$ с условием $z^{\prime}(\zeta)=\sqrt{h(\zeta)}$. В координатах $z$ коэффициент $\widetilde{\Omega}(z)=\Omega(\zeta(z)) \zeta^{\prime 2}(z)$ дифференциала Хопфа имеет $\arg \widetilde{\Omega}=\arg \Omega-2 \arg z^{\prime}(\zeta)=0$, следовательно, $\operatorname{Im} \widetilde{\Omega}=0$, т.е. $\widetilde{\Omega}=\widetilde{L}-\widetilde{N}, \widetilde{M}=0$. Это означает, что поверхность является изотермической ${ }^{6}$. Обратно, из того, что поверхность изотермическая, следует, что $\Delta=0$. Действительно, пусть имеем $\Omega=L-N, g=(L-N)^{2}$, $g_{\bar{\zeta}}=2(L-N) f, g_{\zeta} g_{\bar{\zeta}}=4 g f \bar{f}$. Далее получаем

$$
\begin{aligned}
2 A(L-N) & =2 g[(L-N) f]_{\zeta}-2 f g(L-N)_{\zeta}-4(L-N)^{2} f \bar{f} \\
& =g g_{\zeta \bar{\zeta}}-\frac{3}{2} g_{\zeta} g_{\bar{\zeta}}=B-\frac{1}{2} g_{\zeta} g_{\bar{\zeta}}+2 g f \bar{f}=B,
\end{aligned}
$$

откуда

$$
\operatorname{Im} A=0, \quad 4 g A^{2}-B^{2}=4(L-N)^{2} A^{2}-B^{2}=0 .
$$

Так как при конформном переходе $\widetilde{\zeta}=\widetilde{\zeta}(\zeta)$ к другим координатам $\widetilde{\zeta}$ выражение $\Delta$ изменяется по закону

$$
\widetilde{\Delta}(\widetilde{\zeta})=\Delta(\zeta(\widetilde{\zeta}))\left|\zeta^{\prime}(\widetilde{\zeta})\right|^{20}
$$

равенство $\Delta=0$ остается верным в любой системе изотермических координат.

Поверхности, для которых $\Delta=0$, существуют, причем с выполнением дополнительного условия $A \neq 0$. Такими поверхностями являются, например, поверхности вращения с метрикой вида $d s^{2}=\lambda^{2}(u)\left(d u^{2}+d v^{2}\right)$, для которых ${ }^{7}$ равенство $A=0$ возможно только для специальных меридианов (или для определяемых ими метрических коэффициентов $\lambda(u)$ ), удовлетворяющих некоторому обыкновенному дифференциальному уравнению четвертого порядка, а для остальных поверхностей вращения будет $A \neq 0$. Как мы увидим далее, если при условии $A \equiv 0$ для поверхности существует пара Бонне, то существует и непрерывное семейство парных ей поверхностей Бонне, поэтому предыдущие рассмотрения приводят к следующему дихотомическому свойству изотермических поверхностей.

ТеОрема 3. Изотермическая поверхность локально или не имеет ни одной, или имеет непрерьвное семейство соответствующих ей пар Бонне.

Таким образом, эта теорема является некоторым уточнением теоремы 3.2.1 из [9]. Из теоремы 3, в частности, следует, что ни одна поверхность вращения не может иметь ровно одну парную с ней по Бонне поверхность. И вообще построение конкретных примеров поверхностей, имеющих ровно одну парную с ними по Бонне поверхность, является трудной задачей; теоретическое описание таких поверхностей дано в нескольких работах (см., например, [6]-[9]).

\footnotetext{
${ }^{6}$ По определению поверхность называется изотермической, если существует изотермическая система внутренних координат, в которых коэффициент $M$ равен нулю, т.е. координатные линии являются линиями кривизны.

${ }^{7}$ Возможность введения таких координат подразумевает, что поверхность не содержит полюса.
} 
Рассмотрим более подробно связь изотермических поверхностей с задачей существования пар Бонне. В изотермических координатах (в которых метрика по-прежнему конформно-евклидова, а $M=0$ и $\Omega=L-N)$ и средняя кривизна $H$, и коэффициенты второй формы выражаются через метрический коэффициент $\Lambda$ и его производные. Действительно, в изотермических координатах имеем

$\Omega=L-N, \quad g=(L-N)^{2}=4\left(H^{2}-K\right) \Lambda^{4}, \quad L+N=2 H \Lambda^{2}, \quad L N=K \Lambda^{4}$.

Из этих уравнений получаем

$$
2 H=\frac{L}{\Lambda^{2}}+\frac{K \Lambda^{2}}{L}, \quad 2 H=\frac{N}{\Lambda^{2}}+\frac{K \Lambda^{2}}{N} .
$$

Полагая $\Omega=L-N=L-\frac{K \Lambda^{4}}{L}$, из уравнения (4) получаем

$$
L_{\xi}=\left(\frac{3 L}{\Lambda}-\frac{L^{3}}{K \Lambda^{5}}\right) \Lambda_{\xi}+\left(\frac{K_{\xi}}{K}\right) L, \quad L_{\eta}=\left(\frac{L}{\Lambda}+\frac{K \Lambda^{3}}{L}\right) \Lambda_{\eta} .
$$

Из условия совместности этих уравнений находим уравнение для $L$

$$
a L^{4}+c L^{2}+K^{2} \Lambda^{8} b=0,
$$

где

$$
\begin{aligned}
a & =K \Lambda \Lambda_{\xi \eta}-3 K \Lambda_{\xi} \Lambda_{\eta}-\Lambda K_{\eta} \Lambda_{\xi}, \\
c & =-2 K^{2} \Lambda^{5} \Lambda_{\xi \eta}+6 K^{2} \Lambda^{4} \Lambda_{\xi} \Lambda_{\eta}-\Lambda^{6}\left(K_{\xi \eta} K-K_{\xi} K_{\eta}\right), \\
b & =K \Lambda \Lambda_{\xi \eta}-3 K \Lambda_{\xi} \Lambda_{\eta}-\Lambda K_{\xi} \Lambda_{\eta} .
\end{aligned}
$$

Аналогичным образом, представляя $\Omega$ в виде $\Omega=\frac{K \Lambda^{4}}{N}-N$, получаем уравнение

$$
b N^{4}+c N^{2}+K^{2} \Lambda^{8} a=0,
$$

в котором коэффициенты $a, b, c$ определены, как в формуле $(30)$. Из уравнений (29) и (31) с учетом соотношения $L N=K \Lambda^{4}$ находим

$$
L^{2}=\frac{-c+\varepsilon \sqrt{c^{2}-4 a b K^{2} \Lambda^{8}}}{2 a}, \quad N^{2}=\frac{-c-\varepsilon \sqrt{c^{2}-4 a b K^{2} \Lambda^{8}}}{2 b} .
$$

Но эти значения $L$ и $N$ должны еще удовлетворять уравнениям ПетерсонаКодацци, которые можно представить в виде

$$
\left(L^{2}\right)_{\eta}=\left(\frac{2 L^{2}}{\Lambda}+2 K \Lambda^{3}\right) \Lambda_{\eta}, \quad\left(N^{2}\right)_{\xi}=\left(\frac{2 N^{2}}{\Lambda}+2 K \Lambda^{3}\right) \Lambda_{\xi}
$$

Получается, что и $H$, и $\Omega$ определяются только метрическим коэффициентом $\Lambda$, который должен удовлетворять двум дифференциальным уравнениям пятого порядка. Из этого следует, что такие поверхности должны быть весьма специальными. Но тем не менее они существуют, например, как мы уже отметили выше, они есть среди поверхностей вращения. Выражаемость коэффициентов второй формы через метрику не означает, что изотермичность поверхности является ее внутренним свойством, так как при изгибании поверхности равенство $M=0$ может исчезнуть. 


\section{§ 6. Признак существования единственной пары Бонне}

Рассмотрим теперь случай, когда $C \equiv 0, A \neq 0$ и $\Delta=4 g A \bar{A}-B^{2}>0$. Тогда уравнение (20) имеет два решения (23). Если при этом равенство $D \equiv 0$ не выполнено, то ни одно решение не удовлетворяет уравнению (4), следовательно, нет ни одной поверхности с данными $H$ и $\Lambda$. Если же вместе с $C \equiv 0$ имеем $D \equiv 0$, то непосредственными вычислениями проверяем, что оба решения (23) удовлетворяют уравнению (4), и мы получаем следующую теорему.

Теорема 4. Если заданная метрика (1) и заданная функиия $H$ таковы, что $C \equiv 0, D \neq 0$, то нет ни одной поверхности, имеющей данную метрику u данную среднюю кривизну $H . A$ если $C \equiv 0, D \equiv 0, A \neq 0 u \Delta>0$, то локально существуют ровно две неконгруентные изометричные поверхности с общей средней кривизной и общими омбилическими точками.

Для полноты описания возможных случаев остается рассмотреть ситуацию, когда $A \not \equiv 0$, но есть точки, в которых $A=0$. Мы будем предполагать, что множество $M$, где $A=0$, не имеет внутренних точек (так будет, например, в аналитическом классе поверхностей). Пусть в некоторой области $G \subset V$ имеем $C \equiv 0, D \equiv 0$, и пусть есть множество $M$ меры нуль, $M=\{x \in G \mid A(x)=0\}$, Int $M=\varnothing$, а в его дополнении $A \neq 0$. В точках, где $A \neq 0$, функция $\Omega$ ищется по формуле $(23)$, в которой участвует отношение $\frac{B}{A}$, поведение которого при подходе к точкам множества, вообще говоря, не известно. Если это отношение не продолжимо по непрерывности в точки множества $M$, то в $G$ не существует даже одной поверхности. Поскольку в $G$ есть точки $A \neq 0$, то по предыдущему в $G$ может существовать не более двух поверхностей Бонне. Нас интересует случай существования пары Бонне, и поэтому мы сформулируем следующий признак ее существования.

ТеОрема 5. Для того чтобъ в области $G$ существовали две поверхности, имеющие данную общую метрику и данную общую среднюю кривизну $H$, удовлетворяющие условиям $C \equiv 0, D \equiv 0$, необходимо, а в случае односвязности области и достаточно, чтобы отношение $\frac{B}{A}$ было продолжимо из $G \backslash M$ в $M$ по непрерывности и образовывало в целом в $G$ функцию той же гладкости, что и гладкость $H$.

ДокАЗАТЕЛЬство. Действительно, если есть две поверхности, то разность $\Omega_{2}-\Omega_{1}=\varphi$ определяет в $G$ голоморфную функцию $\varphi$, а отношение $\frac{B}{A}$ равняется в $G \backslash M$ функции $h=\varphi-2 \Omega$, поэтому оно продолжается на всю область как функция, равная $h$.

Обратно, пусть отношение $\frac{B}{A}$ продолжимо в $M$ и образует в $G$ некоторую регулярную функцию $b$. Рассмотрим в $G$ уравнение

$$
\frac{\partial F}{\partial \bar{\zeta}}=\frac{\partial b}{\partial \bar{\zeta}} .
$$

Оно имеет в $G$ регулярные решения $F=b+\Phi$, где $\Phi$ - произвольная голоморфная в $G$ функция. Так как $C \equiv 0$, то в точках из $G \backslash M$ справедливо 
равенство $\left(\frac{B}{2 A}\right)_{\bar{\zeta}}=f$, значит, $\frac{\partial b}{\partial \bar{\zeta}}=f$. Во всех точках из $G \backslash M$ существует функция $\Omega$. Доопределим $\Omega$ в $M$ произвольным образом. Тогда $\Omega$ почти всюду будет иметь производную по $\frac{\partial}{\partial \bar{\zeta}}$, равную $f$, разность $F-\Omega$ будет иметь обобщенную производную по $\frac{\partial}{\partial \bar{\zeta}}$, равную 0 , а в таком случае по соответствующей теореме из [12; гл. 1] эта разность является голоморфной функцией. Значит, функция $\Omega$ может быть определена в $M$ таким образом, что всюду в $G$ имеем $\Omega=F+\psi$, где $\psi$ - голоморфная функция. А тогда определяемая по формуле из $(23)$ функция $\varphi=i \varepsilon \frac{\sqrt{\Delta}}{2 \bar{A}}$, существующая и голоморфная в точках из $G \backslash M$, может быть продолжена как голоморфная на всю область $G$, что и требовалось показать.

\section{§ 7. О продолжении дифференциала Хопфа}

Теперь мы будем предполагать, что на рассматриваемых поверхностях в целом не выполняются неравенства $A \neq 0$ или $C \neq 0$. На самом деле, как это было сказано в $\S 2$, лучше считать, что рассматривается некоторая риманова поверхность $V$, компактная или открытая, а на ней заданы инвариантная относительно конформной замены координат метрическая форма $d s^{2}=\Lambda^{2} d z d \bar{z}$ и числовая функция $H$ и ищется квадратичный дифференциал Хопфа $\Omega d z^{2}$, в каждой карте удовлетворяющий условиям (3), (4). Покажем, как может продолжаться $\Omega$ с малой области на всю поверхность $V$. Пусть значения $\Omega$ в некоторой области $D_{0} \subset V$ в пределах одной карты известны, обозначим ограничение $\Omega$ на $D$ как $\Omega_{0}$. Общее решение уравнения (4) имеет вид (см. [12])

$$
\Omega_{0}(z)=\omega_{0}(z)+\Phi_{0}(z), \quad \omega_{0}(z)=-\frac{1}{\pi} \iint_{D_{0}} \frac{f(\zeta)}{\zeta-z} d \xi d \eta, \quad \zeta=\xi+i \eta
$$

а $\Phi_{0}(z)$ - произвольная голоморфная в $D_{0}$ функция. Мы предполагаем, что нам известна $\Omega_{0}(z)$, значит, нам известна и функция

$$
\Phi_{0}(z)=\Omega_{0}+\frac{1}{\pi} \iint_{D_{0}} \frac{f(\zeta)}{\zeta-z} d \xi d \eta
$$

Впрочем, для $\Phi_{0}(z)$ в [12] дается другой вид

$$
\Phi_{0}=\frac{1}{2 \pi i} \int_{\partial D_{0}} \frac{\Omega_{0}(t)}{t-z} d t
$$

Пусть теперь $D$ - область, содержащая в одной карте внутри себя область $D_{0}$. Значения $\Omega_{D}$ в области $D$ вычисляются по аналогичной (33) формуле

$$
\Omega_{D}(z)=\omega_{D}(z)+\Phi_{D}(z)
$$

Функцию

$$
\omega_{D}(z)=-\frac{1}{\pi} \iint_{D} \frac{f(\zeta)}{\zeta-z} d \xi d \eta
$$


можно представить в виде $\omega_{D}(z)=\omega_{1}(z)+\omega_{2}(z)$, где

$$
\omega_{1}(z)=-\frac{1}{\pi} \iint_{D_{0}} \frac{f(\zeta)}{\zeta-z} d \xi d \eta, \quad \omega_{2}(z)=-\frac{1}{\pi} \iint_{D-D_{0}} \frac{f(\zeta)}{\zeta-z} d \xi d \eta
$$

В точках $z \in D_{0}$ функция $\omega_{2}$ голоморфна, а $\omega_{1}=\omega_{0}$; в тех же точках $\Omega_{D}(z)$ совпадает с $\Omega_{0}(z)$. Следовательно, в этих точках $\Phi_{D}(z)=\Phi_{0}(z)-\omega_{2}(z)$, тем самым, $\Phi_{D}(z)$ для $z \in D-D_{0}$ представляет аналитическое продолжение известной в области $D_{0}$ голоморфной функции. Значит, $\Omega_{D}(z)$ определяется в $D$ единственным образом. Если $D$ и $D_{0}$ не находятся в общей карте, мы найдем сначала представление вида $(33)$ для $\Omega_{0}$ в пересечении $D_{0}$ с $D$ и используем это представление, как выше, для продолжения $\Omega_{0}$ в $D$. Но надо иметь в виду, что если исходить просто из какой-либо функции $\Omega_{0}$ в $D_{0}$, то продолжение ее в более широкую область может не существовать или две разные цепочки продолжений могут привести к разным значениям $\Omega$ в одной и той же карте. Поэтому описанный выше метод продолжения априори предполагает, что $\Omega$ на всей поверхности $V$ уже существует, тогда отправные значения $\Omega_{0}$ в $D_{0}$ окажутся такими, что продолжение на каждом шаге будет возможным и в силу единственности продолжения будут получаться именно значения $\Omega$.

Из сказанного следует, что если $A \neq 0$ хотя бы в одной точке поверхности, то в целом на $V$ может быть не более двух различных дифференциалов Хопфа $\Omega_{1}$ и $\Omega_{2}$. Значит, если на $V$ существует более двух дифференциалов Хопфа, то $A \equiv 0$ на всем $V$.

\section{§ 8. $C^{2}$-гладкие тройки Бонне и семейство Бонне}

Рассмотрим случай $A \equiv 0$ более подробно. Сначала предположим, что на римановой поверхности $V$ с заданными на ней конформно-евклидовой метрикой $d s^{2}=\Lambda^{2}\left(d \xi^{2}+d \eta^{2}\right) \in C^{2}$ и функцией $H \in C$ существуют три дифференциала Хопфа с непрерывными коэффициентами $\Omega_{1}, \Omega_{2}$ и $\Omega_{3}$. Положим

$$
\Omega_{2}-\Omega_{1}=\varphi, \quad \Omega_{3}-\Omega_{1}=\varphi_{1},
$$

где по лемме 1

$$
\varphi d \zeta^{2} \quad \text { и } \varphi_{1} d \zeta^{2}
$$

являются голоморфными квадратичными дифференциалами. Из равенства модулей $\Omega_{j}, 1 \leqslant j \leqslant 3$, имеем уравнения

$$
\bar{\varphi} \Omega_{1}+\varphi \bar{\Omega}_{1}+\varphi \bar{\varphi}=0, \quad \bar{\varphi}_{1} \Omega_{1}+\varphi_{1} \bar{\Omega}_{1}+\varphi_{1} \bar{\varphi}_{1}=0 .
$$

Умножая эти уравнения на $\bar{\Omega}_{1}$, получаем квадратные уравнения

$$
\bar{\varphi} \Omega_{1}^{2}+\varphi \bar{\varphi} \Omega_{1}+g \varphi=0, \quad \bar{\varphi}_{1} \Omega_{1}^{2}+\varphi_{1} \bar{\varphi}_{1} \Omega_{1}+g \varphi_{1}=0 .
$$

Исключая из этих уравнений $g$, получаем

$$
\Omega_{1}=\frac{\varphi \varphi_{1}\left(\bar{\varphi}-\bar{\varphi}_{1}\right)}{\varphi \bar{\varphi}_{1}-\varphi_{1} \bar{\varphi}} .
$$


Теперь находим $g$ через $\varphi$ и $\varphi_{1}$ :

$$
g=-\frac{\left(\varphi \varphi_{1}\right)\left(\bar{\varphi} \bar{\varphi}_{1}\right)\left(\varphi-\varphi_{1}\right)\left(\bar{\varphi}-\bar{\varphi}_{1}\right)}{\left(\varphi \bar{\varphi}_{1}-\bar{\varphi} \varphi_{1}\right)^{2}} .
$$

Так как $\varphi-\varphi_{1}=\Omega_{2}-\Omega_{3}$, то разность $\varphi-\varphi_{1}$ обращается в нуль только в омбилических точках, поэтому числитель в (36) может обращаться в нуль только в омбилических точках, значит, и знаменатель не может быть нулем в неомбилических точках. Покажем, что голоморфные функции $\varphi$ и $\varphi_{1}$ имеют в омбилических точках одинаковый порядок нуля (для случая гладкости класса $C^{\infty}$ это следует из доказанного в $§ 2$ утверждения 1). Действительно, пусть

$$
\varphi \sim a \zeta^{n}, \quad \varphi_{1} \sim b \zeta^{k}, \quad k>n, \quad \zeta \rightarrow 0 .
$$

Тогда

$$
\varphi_{1}=c \varphi \zeta^{k-n} F(\zeta), \quad c=\left(c_{1}+i c_{2}\right) \neq 0, \quad F(0)=1 ;
$$

здесь и далее $F$ является числовой голоморфной функцией. Подставляя эти значения в (36), получаем

$$
g \sim \frac{c \bar{c}(\varphi \bar{\varphi})|\zeta|^{2 k-2 n}}{4\left[\operatorname{Im}\left(c \zeta^{k-n} F\right)\right]^{2}}, \quad \zeta \rightarrow 0 .
$$

В знаменателе имеем гармоническую функцию $\operatorname{Im}\left(c \zeta^{k-n} F(\zeta)\right)$, которая при $k>n$ обращается в нуль не только в точке $\zeta=0$, но и по крайней мере еще на двух кривых, исходящих из точки $\zeta=0$, что невозможно, так как $g-$ непрерывная и ограниченная функция (в данной карте). Значит, $n=k$ (аналогично доказывается невозможность случая $k<n)$. Кроме того, гармоническая функция $\operatorname{Im}(c F(\zeta))$ по-прежнему не может обращаться в нуль в изолированной точке $\zeta=0$, следовательно,

$$
(c F-\bar{c} \bar{F})=2 i \operatorname{Im}(c F(\zeta))=2 i c_{2}+o(1), \quad c_{2} \neq 0,
$$

и как итог получаем, что предполагавшаяся сначала только непрерывной функция

$$
g=-\frac{c \bar{c}(\varphi \bar{\varphi}) F \bar{F}(1-c F)(1-\bar{c} \bar{F})}{(c F-\bar{c} \bar{F})^{2}}
$$

является на самом деле аналитической всюду, включая и возможные омбилические точки. Дополнительно отметим, что функции $F$ и $1-c F$ нигде не обращаются в нуль (так как $\varphi_{1}=c \varphi F$, а равенство $\varphi_{1}=\varphi$ возможно только в омбилических точках, а в них отношение $\frac{\varphi_{1}}{\varphi}$ не стремится к 1$)$.

Используя то же представление $\varphi_{1}$ через $\varphi$, представим $\Omega_{1}$ из (35) в виде

$$
\Omega_{1}=\frac{(\bar{c} \bar{F}-1) c F}{c F-\bar{c} \bar{F}} \varphi,
$$

а вместе с этим находим $\Omega_{2}$ и $\Omega_{3}$ :

$$
\begin{aligned}
\Omega_{2} & =\frac{(c F-1) \bar{c} \bar{F}}{c F-\bar{c} \bar{F}} \varphi, \\
\Omega_{3} & =\frac{(c F-1) c F}{c F-\bar{c} \bar{F}} \varphi .
\end{aligned}
$$


Из этих представлений видим, что все $\Omega_{j}, 1 \leqslant j \leqslant 3$, как функции двух действительных переменных являются аналитическими функциями, значит, существуют $\frac{\partial \Omega_{j}}{\partial \bar{\zeta}}$. Но пока мы не можем сказать, что эти производные равны $f$, так как нам не дано существование производной функции $H$. Предполагая существование $C^{2}$-гладкой поверхности ${ }^{8}$ с данной метрикой класса $C^{2}$ и с данной непрерывной средней кривизной $H$, равенство (8) можно представить в виде (см. [12; гл. 1])

$$
\frac{\partial}{\partial \zeta}\left(T_{D}\left(2 H \Lambda^{2}\right)\right)=-T_{D}\left(4 H \Lambda \Lambda_{z}\right)-\Psi_{D}(\zeta)+\Omega(\zeta)
$$

понимая производную в соболевском смысле. Напомним, что действие оператора $T_{D}$ на произвольную функцию $a(z) \in L_{p}(D), p>2$, определяется по формуле

$$
T_{D}(a)=-\frac{1}{\pi} \iint_{D} \frac{a(z)}{z-\zeta} d z \wedge d \bar{z}
$$

Рассматривая (41) как уравнение для левой части, получаем, что правая часть уравнения принадлежит соболевскому классу $W_{p}^{1}, p>2$, и поэтому его решение

$$
T_{D}\left(2 H \Lambda^{2}\right) \in W_{p}^{2}, \quad 2 H \Lambda^{2}=\frac{\partial}{\partial \bar{\zeta}} T_{D}\left(2 H \Lambda^{2}\right) \in W_{p}^{1},
$$

значит, $H \in W_{p}^{1}$. Зная, что существует производная $H$, мы можем считать, что

$$
\frac{\partial \Omega_{1}}{\partial \bar{\zeta}}=f=2 \Lambda^{2} \frac{\partial H}{\partial \zeta},
$$

а отсюда уже получаем, что $f$ - аналитическая функция, а $H \in C^{2}$. Таким образом, мы доказали следующую теорему.

ТЕОРема 6. Если заданные на римановой поверхности $V$ метрический коэфбициент $\Lambda \in C^{2}$ и непрерывная функиия $H$ таковы, что существуют три поверхности $S_{1}, S_{2}, S_{3}$ класса $C^{2}$ с метрикой $d s^{2}=\Lambda^{2}\left(d \xi^{2}+d \eta^{2}\right)$ и со средней кривизной $H$, то на самом деле функиия $H$ принадлежит $C^{2}$, а определенные по формуле (2) функиии $f, g, \Omega$ являются аналитическими.

Обратимся теперь к вопросу о гладкости самих поверхностей $S_{j}, j=1,2,3$. Сначала исследуем гладкость $\Lambda$. Из определения $g$ и выражения кривизны $K$ через метрический коэффициент $\Lambda$ для $\Lambda$ получаем уравнение

$$
\Delta(\ln \Lambda)=\frac{g-4 \Lambda^{4} H^{2}}{4 \Lambda^{2}} .
$$

Так как правая часть эллиптического уравнения (42) имеет гладкость класса $C^{1, \alpha}$ с любым $0<\alpha<1$, то $\Lambda \in C^{3, \alpha}$, а тогда из равенства

$$
2 \frac{\partial H}{\partial \zeta}=\frac{\partial \Omega_{1}}{\partial \bar{\zeta}} \Lambda^{-2}
$$

\footnotetext{
${ }^{8}$ Никакого противоречия между предполагаемым порядком гладкости поверхности и данным порядком гладкости ее метрики нет, так как если поверхность имеет гладкость класса $C^{n, \alpha}, n \geqslant 2,0<\alpha<1$, или соболевскую $n$-гладкость класса $W_{p}^{n}, n \geqslant 2, p>2$, то ее метрика в изотермических координатах имеет гладкость того же класса (см. [15]); для наших целей достаточно рассматривать гладкость в соболевских производных.
} 
получаем $H \in C^{4, \alpha}$. Возвращаясь к уравнению (42), получаем $\Lambda \in C^{5, \alpha}$, что дает $H \in C^{6, \alpha}$. Повторяя этот процесс, получаем, что функции $\Lambda$ и $H$, а вместе с ними и поверхности $S_{j}, j=1,2,3$, принадлежат классу $C^{\infty}$.

Изучим теперь вопрос об аналитичности $\Lambda$ и $H$ и вместе с этим об аналитичности определяемых ими поверхностей. Для этого из уравнений (3), (4) сначала исключим $H$. Вычисляя $\frac{\partial g}{\partial \zeta}$ и $\frac{\partial^{2} g}{\partial \bar{\zeta} \partial \zeta}$, с учетом уравнения (4) получим

$$
\begin{gathered}
\frac{\partial g}{\partial \zeta}=4 f H e^{2 v}+4(g-4 h) \frac{\partial v}{\partial \zeta}+4 \frac{\partial h}{\partial \zeta} \\
\frac{\partial^{2} g}{\partial \bar{\zeta} \partial \zeta}=2 f \bar{f}+4 H e^{2 v} \frac{\partial f}{\partial \bar{\zeta}}+(g-4 h) \Delta v+E\left(g, g_{\zeta}, g_{\bar{\zeta}}, v_{\zeta}, v_{\bar{\zeta}}, h, h_{\zeta}, h_{\bar{\zeta}}\right)+\Delta h
\end{gathered}
$$

где $v=\ln \Lambda, h=e^{2 v} \Delta v, \Delta$ - оператор Лапласа, а $E$ является некоторой функцией второй степени с постоянными коэффициентами от выписанных при ней аргументов. Если $f \neq 0$, то, умножив (43) на $f_{\bar{\zeta}}$, а (44) на $f$, мы можем из (43) и (44) исключить $H$ и получить для $v$ квазилинейное эллиптическое уравнение четвертого порядка с аналитической зависимостью коэффициентов от независимых переменных, функции $v$ и ее производных. Поэтому, учитывая, что аналитичность $g$ уже доказана, заключаем, что решение $v=\ln \Lambda$ этого уравнения будет вещественно-аналитической функцией, а по формуле $H_{\zeta}=\frac{f}{2 \Lambda^{2}}$ получаем, что и функция $H$ окажется аналитической.

Пусть теперь рассматриваемая точка $p \in V$ является критической точкой для $H$, т.е. $f(p)=0$. Найдем $f$ как производную от $\Omega_{3}$. Имеем

$$
\frac{\partial \Omega_{3}}{\partial \bar{\zeta}}=\frac{c \bar{c} F(c F-1) \varphi \bar{F}^{\prime}}{(c F-\bar{c} \bar{F})^{2}}=f=2 \Lambda^{2} \frac{\partial H}{\partial \zeta} .
$$

Сначала предположим, что точка $p$ не является омбилической. Тогда в ней $f$ имеет нуль только первого порядка. Действительно, нуль функции $f$ будет конечного порядка, так как согласно (45) он одновременно является нулем ${ }^{9}$ голоморфной функции $F^{\prime}(\zeta)$, следовательно, тейлорово разложение $C^{\infty}$-гладкой действительной функции $H$ начинается как

$$
H=H(0)+a_{0} \zeta^{k+1}+a_{1} \zeta^{k} \bar{\zeta}+\cdots+\bar{a}_{1} \zeta \bar{\zeta}^{k}+\bar{a}_{0} \bar{\zeta}^{k+1}+\cdots,
$$

где $k$ - порядок нуля функции $F^{\prime}(\zeta)$ (мы считаем, что точке $p$ в локальной карте соответствует значение $\zeta=0)$. Из $(45)$ для $f$ получим следующее представление:

$$
f=c_{0} \bar{\zeta}^{k}+\cdots, \quad c_{0} \neq 0 .
$$

Сравнивая это выражение с $2 \Lambda^{2} H_{\zeta}$, получаем

$$
c_{0} \bar{\zeta}^{k}+\cdots=2 \Lambda_{0}^{2}\left((k+1) a_{0} \zeta^{k}+k a_{1} \zeta^{k-1} \bar{\zeta}+\cdots+\bar{a}_{1} \bar{\zeta}^{k}\right)+\cdots, \quad|\zeta| \rightarrow 0
$$

что возможно, только если $k=1, a_{1} \neq 0, a_{0}=a_{2}=0$. Представляя $H$ в виде $H=H_{0}+a_{1} \zeta \bar{\zeta}+\cdots$, получаем $\operatorname{Im} a_{1}=0$ и $\operatorname{Im} c_{0}=\operatorname{Im} \frac{\partial f}{\partial \bar{\zeta}}(0)=0$. Значит,

\footnotetext{
9 Для дальнейшего полезно заметить, что верно и обратное: если точка не является критической для средней кривизны, то в ней $F^{\prime} \neq 0$.
} 
коэффициент при $H$ в правой части уравнения (44) в точке $p$ будет действительным и не равным нулю. Тогда после дифференцирования (44) по $\frac{\partial}{\partial \bar{\zeta}}$ и $\frac{\partial}{\partial \zeta}$ и исключения из полученного уравнения $H$ с использованием (44) получаем квазилинейное эллиптическое уравнение шестого порядка относительно $\ln \Lambda$ с аналитическими коэффициентами, тем самым, для рассматриваемого случая тоже получаем аналитичность $\Lambda$ и $H$.

Пусть точка $p$ является омбилической. Тогда в ней голоморфные функции $\varphi(\zeta)$ и $F^{\prime}(\zeta)$ имеют нули соответственно некоторых порядков $n \geqslant 1$ и $k \geqslant 0$. Представляя $f$ и $H$ соответственно как

$$
f=c_{0} \zeta^{n} \bar{\zeta}^{k}+\cdots, \quad H=H(0)+a_{0} \zeta^{n+k+1}+a_{1} \zeta^{n+k} \bar{\zeta}+\cdots+\bar{a}_{0} \bar{\zeta}^{n+k+1}
$$

и сравнивая в (45) левую и правую части, приходим к заключению, что все коэффициенты, кроме $a_{k}$ и $a_{n+1}=\bar{a}_{k}$, равны нулю. Тогда получаем равенство

$$
c_{0} \zeta^{n} \bar{\zeta}^{k}+\cdots=2 \Lambda_{0}^{2}\left((n+1) a_{k} \zeta^{n} \bar{\zeta}^{k}+k a_{n+1} \zeta_{k-1} \bar{\zeta}^{n+1}\right)
$$

которое выполнимо лишь ${ }^{10}$ при $k=n+1$ и $c_{0}=2 \Lambda_{0}^{2} k a_{k}$ с $\operatorname{Im} a_{k}=0$. Следовательно, коэффициент при $H$ в правой части уравнения (44) эквивалентен $a \zeta^{n} \bar{\zeta}^{n}, a \neq 0, \operatorname{Im} a=0$. После дифференцирования этого уравнения $n$ раз по $\frac{\partial}{\partial \bar{\zeta}}$ и $n$ раз по $\frac{\partial}{\partial \zeta}$ получится уравнение, в которое $H$ войдет с отличным от нуля и действительным в точке $p$ коэффициентом. Если затем продифференцировать это уравнение по $\frac{\partial}{\partial \bar{\zeta}}$ и $\frac{\partial}{\partial \zeta}$ и с помощью исходного уравнения (44) исключить из полученного уравнения $H$, то для $v$ получим квазилинейное эллиптическое уравнение порядка $2 n+6$ с аналитическими коэффициентами. Значит, функции $\Lambda$ и $H$, а вместе с ними и поверхности $S_{j}, j=1,2,3$, являются аналитическими.

Далее, получим представление через $\Omega_{1}, \Omega_{2}$ и $\Omega_{3}$ всех решений системы $(3),(4)$. Непосредственно проверяется, что если функция $\Omega_{1}$ (а вместе с ней и $\left.\Omega_{2}\right)$ удовлетворяет уравнению (4), то функция

$$
\Omega(\zeta, t)=\frac{\Omega_{1}\left(\varphi_{1}-\varphi\right) \cos t+\Omega_{2} \varphi_{1} \sin t}{\left(\varphi_{1}-\varphi\right) \cos t+\varphi_{1} \sin t}
$$

(где параметр $t$ - действительное число, $0 \leqslant t \leqslant \pi$ ) тоже удовлетворяет уравнению (4). Для проверки (3) достаточно представить $\Omega(\zeta, t)$ с учетом формул (38)-(40) в виде

$$
\Omega(\zeta, t)=\Omega_{3} \frac{(\bar{c} \bar{F}-1) \cos t+\bar{c} \bar{F} \sin t}{(c F-1) \cos t+c F \sin t} .
$$

Отметим, что знаменатель в формуле (46) обращается в нуль только в омбилических точках. Действительно, если знаменатель равен нулю, то равен нулю и числитель, а тогда для $\cos t$ и $\sin t$ имеем однородную систему, которая в неомбилических точках может иметь только нулевое решение.

10 Тем самым, мы получили "прямое" и более простое, чем в [9], доказательство лемм 3.3.1 и 3.3 .2 из этой работы, причем без априорного предположения гладкости семейства Бонне относительно параметра семейства. 
Теперь нужно доказать, что формула (46) дает общее решение системы (3), (4). Пусть $\Omega_{0}-$ некоторое ее решение. Положив $\Omega_{0}-\Omega_{1}=\varphi_{0}$, мы, как в начале этого параграфа, получим следующие представления решений:

$$
\begin{gathered}
\Omega_{1}=\frac{\left(\bar{c}_{0} \bar{F}_{0}-1\right) c_{0} F_{0}}{c_{0} F_{0}-\bar{c}_{0} \bar{F}_{0}} \varphi, \quad \Omega_{2}=\frac{\left(c_{0} F_{0}-1\right) \bar{c}_{0} \bar{F}_{0}}{c_{0} F_{0}-\bar{c}_{0} \bar{F}_{0}} \varphi \\
\Omega_{0}=\frac{\left(c_{0} F_{0}-1\right) c_{0} F_{0}}{c_{0} F_{0}-\bar{c}_{0} \bar{F}_{0}} \varphi
\end{gathered}
$$

где по-прежнему $\varphi=\Omega_{2}-\Omega_{1}, \varphi_{0}=\Omega_{0}-\Omega_{1}, \varphi_{0}=c_{0} \varphi F_{0}, F_{0}(0)=1$.

Найдем связь между голоморфными функциями $F(\zeta)$ и $F_{0}(\zeta)$. Для этого найдем $f$ как производную функции $\Omega_{3}$, взятой в виде $(40)$. Имеем

$$
\frac{\partial \Omega_{3}}{\partial \bar{\zeta}}=\frac{c \bar{c} F(c F-1) \varphi \bar{F}^{\prime}}{(c F-\bar{c} \bar{F})^{2}}=f .
$$

Из этого уравнения и из (37) получаем

$$
f=g \bar{\Phi}
$$

где $\Phi=\frac{F^{\prime}}{(1-c F) \varphi F}$. Выше при доказательстве аналитичности функций $\Lambda$ и $H$ было установлено, что в омбилических точках порядок $k$ нуля функции $F^{\prime}$ связан с порядком $n$ нуля $\varphi$ равенством $k=n+1$, поэтому функция $\Phi$ всюду (в пределах одной карты) является голоморфной. Соотношение (49) можно представить как уравнение

$$
F^{\prime}=F(1-c F) \omega, \quad \omega=\frac{\varphi \bar{f}}{g},
$$

для $F$, так как $\omega$ зависит только от известных функций $f, g$ и $\varphi$. Аналогичное уравнение

$$
F_{0}^{\prime}=F_{0}\left(1-c_{0} F_{0}\right) \omega
$$

можно использовать для нахождения функции $F_{0}$ (начальное условие $F_{0}(0)=1$ известно по определению самой функции). Заменяя в (51) коэффициент $\omega$ его значением из (50), получаем легко интегрируемое уравнение

$$
\frac{F_{0}^{\prime}}{1-c_{0} F_{0}}=\frac{F^{\prime}}{1-c F},
$$

из которого имеем

$$
F_{0}=\frac{(1-c) F}{1-c_{0}+\left(c_{0}-c\right) F} .
$$

Для нахождения $c_{0}$ воспользуемся тем, что функция $\Omega_{1}$ имеет два представления: $(38)$ и в $(47)$. Подставим в $(47)$ значение $F_{0}$ из $(52)$ и сравним полученное выражение с (38). Получим, что коэффициент $c_{0}$ должен удовлетворять уравнению

$$
c_{0}(1-c)\left(\bar{c}_{0}-\bar{c}\right)-\bar{c}_{0}\left(c_{0}-c\right)(1-\bar{c})=0
$$


Пусть $c_{0}=R e^{i \theta}$ (как и $c$, коэффициент $c_{0}$ не равен нулю), откуда, используя (53), находим $R$ :

$$
R=\frac{\bar{c}(1-c) e^{i \theta}-c(1-\bar{c}) e^{-i \theta}}{\bar{c}-c} .
$$

Далее с учетом (52) представим $\Omega_{0}$ в виде

$$
\Omega_{1}+\varphi_{0}=\Omega_{1}+c_{0} F_{0} \varphi=\Omega_{1}+\frac{c_{0}(1-c) F}{1-c_{0}+\left(c_{0}-c\right) F} \varphi
$$

а формулу (46) преобразуем к виду

$$
\Omega(\zeta, t)=\Omega_{1}+\frac{c F \sin t}{(c F-1) \cos t+c F \sin t} \varphi .
$$

Равенство $\Omega_{0}$ значению $\Omega(\zeta, t)$ при некотором $t=t_{0}$ равносильно выполнению соотношения

$$
\frac{c F \sin t_{0}}{(c F-1) \cos t_{0}+c F \sin t_{0}}=\frac{c_{0}(1-c) F}{1-c_{0}+\left(c_{0}-c\right) F},
$$

которое выполнено при $\operatorname{ctg} t_{0}=\frac{c\left(c_{0}-1\right)}{c_{0}(1-c)}$. Учитывая, что величина $\left|c_{0}\right|=R$ дается формулой ${ }^{11}(54)$, получаем искомое значение $t_{0}$ :

$$
t_{0}=\frac{\pi}{2}+\operatorname{arcctg} \frac{c \bar{c} \sin \theta}{\operatorname{Im}\left(\bar{c}(c-1) e^{i \theta}\right)} .
$$

Как итог этого параграфа получаем следующую теорему

Теорема 7. Если есть три $C^{2}$-гладкие гомеоморфные диску изометричные поверхности с общей средней кривизной $H$, то таких поверхностей существует бесчисленное множество и все они составляют гомеоморфное окружности однопараметрическое и аналитическое по параметру семейство поверхностей, коэфбициенты второй квадратичной формы которых задаются формулами (5) и (46), причем все поверхности семейства, включая исходные, являются аналитическими.

Итак, теорема 7 дает полное описание всего множества решений системы $(3),(4)$, или конфигурационного пространства решений задачи изометрического погружения данной метрики с предписанной средней кривизной искомых поверхностей. Гомеоморфность множества решений окружности означает, что конфигурационное пространство поверхности при ее изгибании с сохранением средней кривизны параметризуется точками окружности, так что деформируемая поверхность в ходе изгибания возвращается в исходное положение, проходя через все неконгруентные между собой ее формы.

Отметим также, что обычно рассмотрение семейств пар Бонне проводится при априорном предположении, что имеется дифференцируемая зависимость

\footnotetext{
11 Это и гарантирует, что рассматриваемое значение котангенса является вещественным числом.
} 
семейств от параметра, а в теореме 7 установлено, что если семейство поверхностей существует (а для его наличия достаточно существование трех поверхностей Бонне - факт, известный еще Картану, но в предположении априорной аналитичности поверхностей; см. [2]), то оно обязательно имеет аналитическую зависимость от параметра. Кроме того доказана аналитичность всех поверхностей семейства, включая и возможные критические точки средней кривизны (существование таких поверхностей показано в [9; с. 51]).

\section{§9. Метрика поверхностей семейства Бонне}

Известно, что метрики поверхностей семейства Бонне являются метриками вращения (см., например, [2], [5], [9]). Это свойство имеет важное значение при рассмотрении поверхностей Бонне "в целом". Мы дадим новое его доказательство в едином изложении для окрестностей точек всех видов - и омбилических, и критических для $H$, но не омбилических, и некритических для $H$.

ТеОрема 8. Метрики поверхностей семейства Бонне являются метриками вращения.

Напомним (см. [16]), что метрика называется метрикой вращения или метрикой с локальной круговой симметрией, если ее можно представить в виде $d s^{2}=\Lambda^{2}(r)\left(d u^{2}+d v^{2}\right)$, где $r^{2}=u^{2}+v^{2}$.

ДоКАЗАТЕЛЬСТво ТЕОРЕмЫ 8. В ходе доказательства теоремы 7 с использованием формулы (45) было установлено, что, во-первых, критические точки средней кривизны изолированные, во-вторых, в критической точке $p$ средней кривизны производная $F^{\prime}(p)$ имеет нуль порядка $k=n+1$, где $n \geqslant 0$ - порядок нуля разности дифференциалов Хопфа, т.е. $n=0$, если точка $p$ неомбилическая, и $n \geqslant 1$, если точка $p$ омбилическая (и $F^{\prime}(p) \neq 0$, если точка $p$ некритическая для $H$ ). При этом было показано, что

$$
f=\frac{c \bar{c} F(c F-1) \varphi \bar{F}^{\prime}}{(c F-\bar{c} \bar{F})^{2}} \sim c_{0} \zeta^{n} \bar{\zeta}^{n+1}, \quad \operatorname{Im} c_{0} \neq 0, \quad n \geqslant 0
$$

(из этой формулы видно, что каждая омбилическая точка является критической для $H$, но обратное неверно). Представим формулу (45) в виде

$$
\frac{\bar{F}^{\prime}(\zeta)}{h(\zeta)(c F-\bar{c} \bar{F})^{2}}=2 \Lambda^{2} \frac{\partial H}{\partial \zeta}
$$

где $h=\frac{1}{c \bar{c}(c F-1) \varphi}$. Умножая $(57)$ на голоморфную функцию $h F^{\prime}$, получаем соотношение

$$
\frac{F^{\prime} \bar{F}^{\prime}}{(c F-\bar{c} \bar{F})^{2}}=2 \Lambda^{2} \frac{\partial H}{\partial \zeta} h F^{\prime}(\zeta)
$$

откуда

$$
\operatorname{Im}\left(\frac{\partial H}{\partial \zeta} h F^{\prime}(\zeta)\right)=0
$$


С учетом соотношения (56) получаем

$$
h(\zeta) F^{\prime}(\zeta) \sim c_{1} \zeta, \quad c_{1}=-\frac{b \bar{b}}{4(\operatorname{Im}(c F))^{2} c_{0}} \neq 0, \quad \operatorname{Im} c_{1}=0
$$

где число $b \neq 0$ определяется соотношением $F^{\prime}(\zeta) \sim b \zeta^{n+1}, \zeta \rightarrow 0$. Введем новую переменную $w=u+i v$ по формуле

$$
\ln w=c_{1} \int \frac{1}{h F^{\prime}} d \zeta
$$

которая изменяется по закону $w=\zeta+\cdots$, если точка $p$ критическая для функции $H$ со значением координаты $\zeta(p)=0$, и по закону $w=w_{0}+\zeta+\cdots$, где $w_{0} \neq 0$, если точка $p$ не является для $H$ критической. После такой замены уравнение (58) примет вид

$$
\operatorname{Im}\left(\frac{\partial H}{\partial w} w\right)=0
$$

и его решение $H$ будет функцией от одной переменной $t=u^{2}+v^{2}$ (так как из уравнения следует, что производная $H$ в угловом направлении равна нулю, а зависимость функции $H$ только от квадрата расстояния вытекает из ее аналитичности). Теперь покажем, что и метрический коэффициент $\Lambda^{2}$ в координатах $(u, v)$ будет функцией от $t$. Для этого обратимся к формуле (48), предполагая, что мы работаем во введенных выше координатах. Перепишем эту формулу в виде

$$
\frac{\partial \Omega_{3}}{\partial \bar{w}}=\frac{c \bar{c} F(c F-1) \varphi \bar{F}^{\prime}}{(c F-\bar{c} \bar{F})^{2}}=2 \Lambda^{2} H^{\prime}(t) \bar{w} .
$$

Обозначим через $q(w)$ голоморфную функцию $c F(c F-1) \varphi$. Тогда соотношение (60) можно представить в виде

$$
\frac{q(w) w}{c F^{\prime}}=-8 \Lambda^{2} H^{\prime} w \bar{w} \frac{(\operatorname{Im}(c F))^{2}}{c \bar{c} F^{\prime} \bar{F}^{\prime}} .
$$

Левая часть этого равенства является голоморфной функцией, а правая часть действительная функция. Следовательно, левая часть является некоторой действительной постоянной $c_{2}$, т.е. $q(w) w=c c_{2} F^{\prime}(w)$. Так как по формуле $(40)$

$$
\Omega_{3}=\frac{q(w)}{c F-\bar{c} \bar{F}}
$$

то формулу (60) можно переписать следующим образом:

$$
\frac{\partial \Omega_{3}}{\partial \bar{w}}=\Omega_{3} \frac{\bar{c} \bar{F}^{\prime}}{c F-\bar{c} \bar{F}}=2 \Lambda^{2} H^{\prime} \bar{w}
$$

откуда

$$
\Omega_{3} \bar{\Omega}_{3} \frac{c \bar{c} F^{\prime} \bar{F}^{\prime}}{(c F-\bar{c} \bar{F})(\bar{c} \bar{F}-c F)}=4 \Lambda^{4}{H^{\prime}}^{2} w \bar{w}
$$


С другой стороны, подставляя в (62) значение $\Omega_{3}$ из (61) и учитывая вид функции $q(w)$, получаем

$$
\frac{c \bar{c} c_{2} F^{\prime} \bar{F}^{\prime}}{w(c F-\bar{c} \bar{F})^{2}}=2 \Lambda^{2} \bar{w} H^{\prime}
$$

откуда с учетом уравнения (63) получаем

$$
g=\Omega_{3} \bar{\Omega}_{3}=4\left(H^{2}-K\right) \Lambda^{4}=-2 c_{2} \Lambda^{2} H^{\prime}(r)>0, \quad r=w \bar{w} .
$$

Теперь вспомним, что введенная в (18) величина

$$
B=g \frac{\partial^{2} g}{\partial \zeta \partial \bar{\zeta}}-\frac{\partial g}{\partial \zeta} \frac{\partial g}{\partial \bar{\zeta}}-2 g f \bar{f}=g^{2} \frac{\partial^{2} \ln g}{\partial \zeta \partial \bar{\zeta}}-2 g f \bar{f}
$$

равна нулю, поэтому, подставив сюда значение $g$ из (64), с учетом уравнения Гаусса $\Delta \ln \Lambda=-K \Lambda^{2}$ после несложных преобразований получаем

$$
\left(t \frac{H^{\prime \prime}(t)}{H^{\prime}(t)}\right)_{t}^{\prime}-\frac{c_{2}}{4} H^{\prime}(t)+\Lambda^{2}\left(\frac{4 t}{c_{2}} H^{\prime}(t)-\frac{H^{2}(t)}{2}\right)=0
$$

откуда видно, что $\Lambda^{2}$ зависит только от $t=w \bar{w}$, что и доказывает теорему.

Более подробное описание структуры функции $H(t)$ и метрического коэффициента $\Lambda$ можно найти в [9].

\section{§ 10. Признак изгибаемости поверхности с сохранением средней кривизны}

Сказанное в $\S 8$ было справедливо в предположении существования трех поверхностей Бонне, что влечет существование уже непрерывного семейства Бонне. В этом параграфе мы приведем условия на функцию средней кривизны и на метрику, гарантирующие существование трех поверхностей Бонне, а значит, и семейства Бонне. Как мы знаем (см. конец $\S 7$ ), если $A \neq 0$, то может существовать не более двух поверхностей. Следовательно, для существования трех и более поверхностей необходимо, чтобы было $A \equiv 0$. Более того, необходима также аналитичность поверхности и представимость $f$ согласно (49) в виде

$$
f=g \bar{\Phi}(\zeta)
$$

где $\Phi(\zeta)$ - голоморфная функция ${ }^{12}$.

\footnotetext{
${ }^{12}$ На самом деле из условия $A \equiv 0$ вместе с $\left(\frac{g}{f}\right)_{\zeta}=0$ можно также вывести, что $\left(\frac{g}{f}\right)_{\zeta}=0$, т.е. что $g=f \bar{\Psi}(\zeta)$, где $\Psi d \zeta$ - не обязательно голоморфный, а, возможно, мероморфный дифференциал, и это даже более точно описывает ситуацию в том смысле, что с помощью $\Phi(\zeta)$ нельзя образовать голоморфный дифференциал, инвариантный относительно конформных замен координат, а такой дифференциал получается для функции $\Psi=\frac{1}{\Phi}$, только он будет теперь уже мероморфным дифференциалом. Предположение мероморфности $\Psi$ объясняется тем, что мы заранее не знаем соотношение нулей $f$ и $g$, а голоморфность $\Phi$ нам известна как необходимое условие существования тройки поверхностей Бонне.
} 
Покажем, что при выполнении только условия (49) верна следующая

ТеОрема 9. Любая односвязная область $D$ на поверхности $S$ гладкости $C^{3}$ с выполнением условия (49) допускает изгибания в классе поверхностей Бонне.

ДокАзАтельство. Найдем изометричные $D$ поверхности с той же средней кривизной, что и у $S$. Для этого достаточно найти дифференциалы Хопфа $\omega d \zeta^{2}=(l-n-2 i m) d \zeta^{2}$, удовлетворяющие уравнениям (3), (4). Будем искать такие функции в виде $\omega=\Omega e^{i \theta}$. Имеем уравнение

$$
\frac{\partial \Omega}{\partial \bar{\zeta}}=f e^{i \theta}+i \Omega e^{i \theta} \frac{\partial \theta}{\partial \bar{\zeta}}=f,
$$

из которого с использованием условия (49) находим, что, во-первых, функция $\theta$ должна быть гармонической (подробные вычисления см. в § 12), во-вторых, она однозначно определяется из уравнения

$$
\theta_{\bar{\zeta}}=i \bar{\Phi}\left(1-e^{-i \theta}\right) \bar{\Omega}
$$

если задать ее значения в некоторой одной точке. Эти значения и будут играть роль параметра семейства Бонне. Так как формы $\Omega$ и $\omega$ по утверждению 1 не могут быть эквивалентными при подходе к любой данной точке, то при выборе в данной точке значений $\theta$ между 0 и $2 \pi$ получаем формы $\omega$, отличные от $\Omega$, а значениям $\theta=0$ и $\theta=2 \pi$ соответствуют решения $\theta \equiv 0$ или $\omega=\Omega$, т.е. множество решений гомеоморфно окружности, что и согласуется с теоремой 6 .

Мы знаем, что при наличии тройки Бонне соответствующие поверхности являются аналитическими и на них выполнено представление (49). Поэтому только что доказанную теорему можно сформулировать в следующей более завершенной форме.

Теорема 10. Для того чтобы односвязная поверхность изгибалась с сохранением средней кривизны, необходимо и достаточно, чтобы она была аналитической и чтобы на ней отношение $\frac{\bar{f}}{g}$ было голоморфной функцией.

ЗАмечАниЕ 10. Таким образом, мы имеем критерий принадлежности поверхности семейству Бонне, выраженный в терминах ее метрики и функции средней кривизны. В литературе есть достаточно полное описание таких поверхностей с точки зрения их внешнего строения (см. §1).

ЗАмЕчАниЕ 11. Нам представляется, что более эффективным критерием принадлежности поверхности семейству Бонне было бы утверждение, что для этого необходимо и достаточно выполнение равенства $A=g f_{\zeta}-f g_{\zeta} \equiv 0$, так как для прямой проверки справедливости представления (49) сначала нужно убедиться, что $A=0$. Но это равенство является только необходимым условием, поэтому интересной и пока нерешенной задачей является доказательство или опровержение достаточности равенства $A=0$ для наличия представления $(49)$ с голоморфным множителем $\Phi(\zeta)$. Проблема здесь в том, что мы заранее не знаем, регулярна ли функция $\frac{f}{g}$. 


\section{$\S 11$. Теоремы о парах и тройках Бонне для компактных $C^{2}$-гладких поверхностей}

До сих пор мы занимались теорией поверхностей Бонне в локальной постановке, или, более общо, в случае односвязных поверхностей. Далее мы хотим снять это ограничение и рассматривать компактные поверхности (без края), о некоторых результатах для которых говорилось в $\S 1$. Мы там упоминали о проблеме существования пар Бонне среди компактных поверхностей. Эта проблема состоит в доказательстве существования или несуществования двух изометричных поверхностей в предположении совпадения их средних кривизн. Напомним, что в теории изгибаний компактных поверхностей без всякого априорного предположения обнаружена связь между средними кривизнами поверхностей, возникающих в процессе непрерывных изометрических деформаций, а именно, в [17; с. 676] отмечен факт инвариантности интегральной средней кривизны поверхности при ее изгибаниях ${ }^{13}$. Доказательству этого результата и его инфинитезимального аналога посвящено несколько статей (см. [18]-[20]), но так как ни в одной из этих публикаций нет ссылки на работу [17], а в самой работе [17] это свойство изгибаемых поверхностей приводится автором без доказательства, мы сочли возможным привести здесь простое его доказательство $^{14}$.

Для доказательства мы используем известную формулу Герглотца, причем приведем ее в обобщенном виде, полученном в [21]:

$$
\begin{gathered}
2 \iint_{S} h H d A-2 \iint_{S} h H^{*} d A+\iint_{S} \frac{p h}{\Lambda^{4}}\left|\begin{array}{cc}
L-L^{*} & M-M^{*} \\
M-M^{*} & N-N^{*}
\end{array}\right| d A \\
=\iint_{S}\left[\left(\mathbf{r}, \mathbf{r}_{\xi}\right) \frac{\left(M-M^{*}\right) h_{\eta}-\left(N-N^{*}\right) h_{\xi}}{\Lambda^{4}}\right. \\
\left.+\left(\mathbf{r}, \mathbf{r}_{\eta}\right) \frac{\left(M-M^{*}\right) h_{\xi}-\left(L-L^{*}\right) h_{\eta}}{\Lambda^{4}}\right] d A,
\end{gathered}
$$

где $h(\xi, \eta)$ - произвольная $C^{1}$-гладкая функция на $C^{2}$ - гладкой компактной поверхности $S$, а $\mathbf{r}$ - ее радиус-вектор, $p=(\mathbf{r}, \mathbf{n})$ - опорная функция поверхности, $L^{*}, M^{*}, N^{*}, H^{*}$ - коэффициенты второй формы и средняя кривизна некоторой поверхности $S^{*}$, изометричной $S$. Если функция $h$ равна 1 , приходим к известной формуле Герглотца ${ }^{15}$

$$
\begin{aligned}
& 2 \iint_{S} H\left(t_{2}\right) d A-2 \iint_{S} H\left(t_{1}\right) d A \\
& \quad=\iint_{S} \frac{1}{\Lambda^{4}} \begin{array}{cc}
L\left(t_{2}\right)-L\left(t_{1}\right) & M\left(t_{2}\right)-M\left(t_{1}\right) \\
M\left(t_{2}\right)-M\left(t_{1}\right) & N\left(t_{2}\right)-N\left(t_{1}\right)
\end{array} \mid p\left(t_{1}\right) d A,
\end{aligned}
$$

\footnotetext{
${ }^{13}$ На самом деле это утверждение пока является чисто гипотетическим, так как еще нет ни одного примера компактной изгибаемой поверхности класса гладкости $C^{2}$.

${ }^{14}$ Нужно признаться, занимаясь проблемой инвариантности интегральной средней кривизны, мы тоже вначале не заметили, что этот результат уже был объявлен в [17], причем оказалось, что мы использовали ровно тот подход, что и был предложен в [17].

${ }^{15}$ Одно из ее доказательств в классе гладкости $C^{3}$ можно найти, например, в работе [22].
} 
где аргументы $t_{1}$ и $t_{2}$ соответствуют двум поверхностям из семейства изгибания. Пусть изгибания происходят в классе гладкости $C_{2,1}^{2}$ (это значит, что поверхности в ходе изгибания остаются в классе $C^{2}$, а их радиусы-векторы вместе с производными до второго порядка включительно однократно непрерывно дифференцируемы по параметру деформации $t$ ). Обозначим интегральную среднюю кривизну как $J(t)$. Так как все участвующие в формуле величины дифференцируемы по $t$, то из нее получаем оценку

$$
\left|J\left(t_{2}\right)-J\left(t_{1}\right)\right| \leqslant C\left|t_{2}-t_{1}\right|^{2},
$$

откуда и следует, что

$$
J(t)=\iint_{S} H(t) d A=\text { const. }
$$

Заметим, что на самом деле для справедливости утверждения достаточно предположить, что зависимость коэффициентов вторых квадратичных форм от параметра деформации имеет гладкость класса $C^{0, \alpha}, \alpha>\frac{1}{2}$.

Теперь покажем, как можно просто получить информацию о количестве омбилических точек на компактной поверхности, если для нее есть пара Бонне. Известно, что если на некоторой компактной римановой поверхности дан мероморфный дифференциал $\varphi_{0} d \zeta$ первого порядка, то числа $N_{0}$ и $P_{0}$ соответственно его нулей и полюсов с учетом их кратности связаны равенством $N_{0}-P_{0}=2 p-2$, где $p$ - топологический род поверхности (см. [23]). В случае существования пары Бонне на поверхности есть голоморфный квадратичный дифференциал $\varphi d \zeta^{2}$. Возьмем на поверхности некоторый мероморфный дифференциал $\varphi_{0} d \zeta$ (существование его известно). Тогда отношение $\frac{\varphi d \zeta^{2}}{\varphi_{0} d \zeta}$ будет некоторым мероморфным дифференциалом $\varphi_{1} d \zeta$. Пусть $N, P$ и $N_{1}, P_{1}-$ числа нулей и полюсов в дифференциалах $\varphi d \zeta^{2}$ и $\varphi_{1} d \zeta$ соответственно. Для дифференциала $\varphi_{1} d \zeta$ имеем $N_{1}=N+P_{0}, P_{1}=P+N_{0}$, поэтому

$$
N_{1}-P_{1}=N-P+\left(P_{0}-N_{0}\right)=N-P+(2-2 p)=2 p-2,
$$

значит, $N-P=4 p-4$. Для нашего голоморфного квадратичного дифференциала $\varphi d \zeta^{2}$ полюсов нет, следовательно,

$$
N=4 p-4 .
$$

Теперь вспомним, что главные направления $d \eta: d \xi$ являются корнями уравнения

$$
M(d \eta)^{2}+(L-N) d \xi d \eta-M(d \xi)^{2}=0,
$$

поэтому сумма углов этих направлений по отношению к некоторому фиксированному направлению в локальной карте дает угол $\alpha$, для которого имеем равенство $\operatorname{tg} \alpha=-\operatorname{tg}(\arg \bar{\Omega})$. Каждое главное направление при обходе омбилической точки дает приращение угла, кратное $\pi$, а суммарное их приращение получается кратным $2 \pi$ и равным приращению аргумента $\bar{\Omega}$. В свою очередь приращение этого аргумента (см. формулу (11)) совпадает с приращением аргумента функции $\bar{\varphi}$, следовательно, порядок нуля функции $\varphi$ кратен с коэффициентом -2 индексу поля направлений, задаваемых касательными прямыми 
к линиям кривизны. В частности, это значит, что индексы всех омбилических точек на парах Бонне отрицательны, и если нуль функции $\varphi$ простой, то индекс линейного поля направлений будет равен $-\frac{1}{2}$, т.е. при попытке ввести на линиях кривизны касательные векторы они при обходе вокруг омбилической точки вернутся в исходную точку с противоположными направлениями, повернувшись на угол $-\pi$. Таким образом, на парах Бонне рода $p$ бывает самое большее $4 p-4$ омбилических точек с общим индексом $2-2 p$, откуда, в частности, следует, что пар Бонне среди поверхностей рода 0 не бывает, а в случае поверхностей рода 1 на них вовсе не будет ни одной омбилической точки ${ }^{16}$.

Теперь приведем новые простые доказательства известного в литературе результата об отсутствии пар Бонне для поверхностей, гомеоморфных сфере, и троек Бонне для поверхностей рода $p \geqslant 1$, но предполагая при этом минимально возможную гладкость рассматриваемых поверхностей, а именно, что класс гладкости $C^{2}$.

ТеОрема 11. Среди компактных поверхностей класса гладкости $C^{2}$ и рода $p=0$ нет пар Бонне.

ТеОрема 12. Среди компактных поверхностей класса гладкости $C^{2}$, рода $p \geqslant 1$ и непостоянной средней кривизны нет троек Бонне.

ДокАЗАТЕЛЬСтво тЕоремы 11. Первое доказательство с применением формулы (66) уже было дано выше: сфера имеет род $p=0$, поэтому в случае существования пары Бонне число нулей голоморфного дифференциала оказывается равным отрицательному числу, что невозможно. Для второго доказательства используем возможность введения на гомеоморфной сфере поверхности с одной выколотой точкой изотермических координат $\zeta=\xi+i \eta$ единственной картой, в которой разность двух дифференциалов Хопфа будет аналитической функцией $\varphi(\zeta)$ на всей открытой комплексной плоскости. Введя в окрестности выколотой точки регулярные координаты $\widetilde{\zeta}=\frac{1}{\zeta}, \widetilde{\zeta} \rightarrow 0$ при $\zeta \rightarrow \infty$, мы получим, что функция $\varphi(\zeta)$ связана с новой функцией $\widetilde{\varphi}(\widetilde{\zeta})$ равенством $\varphi(\zeta)=-\widetilde{\varphi}(\widetilde{\zeta}(\zeta)) \zeta^{-2} \rightarrow 0, \zeta \rightarrow \infty$, следовательно, $\varphi \equiv 0$ и двух неконгруэнтных поверхностей Бонне нет.

ДоКАЗАТЕЛЬСтво тЕОРЕмЫ 12. Пусть есть три поверхности Бонне. При доказательстве теоремы 7 мы вводили нигде не равную нулю голоморфную функцию $F(\zeta)$, которая на компактной римановой поверхности должна быть постоянной. Там же мы вывели формулу (48), в которой в качестве множителя участвовала производная от $F$, равенство нулю которой приводит к тождественному равенству нулю производных от $H$.

Второе доказательство теоремы 12 для поверхностей рода $p>1$ состоит в следующем. Мы знаем, что существование тройки Бонне локально приводит

\footnotetext{
${ }^{16}$ Конечно, классическая формула Ind $=\chi=2-2 p$, где $\chi$ - эйлерова характеристика компактной поверхности, справедлива для любого поля направлений с изолированными особенностями, как и для поля векторов, но, во-первых, на эту формулу в литературе практически не обращают внимание (мне ее доказательство сообщил А. А. Гайфуллин, которому я, пользуясь случаем, выражаю свою искреннюю благодарность), во-вторых, из общей формулы вовсе не следует, что индексы омбилических точек должны быть отрицательными.
} 
к существованию семейства Бонне, а согласно теореме 8 метрики поверхностей семейства Бонне являются аналитическими метриками вращения. А в работе [16] показано, что аналитические метрики вращения могут быть только на сфере и торе.

\section{§ 12. Некоторые необходимые/достаточные условия несуществования компактных пар Бонне}

Перейдем к вопросу о существовании/несуществованию среди компактных поверхностей пар Бонне. $\mathrm{K}$ сожалению, в доказательстве анонсированного нами ранее в [24] результата об отсутствии таких пар Бонне обнаружилась ошибка, поэтому рассмотрим здесь только некоторые необходимые признаки существования или достаточные признаки отсутствия пар Бонне. Начнем со следующего признака.

Теорема 13. Если на компактной $C^{4}$-гладкой поверхности любого рода и c непостоянной средней кривизной всюду $A=g f_{\zeta}-f g_{\zeta}=0$, то для этой поверхности пары Бонне нет.

ДокАзАтельство. Допустим, что для данной поверхности $S_{1}$ существует парная ей по Бонне поверхность $S_{2}$. Как известно из $\S 2$, тогда существует функция $\theta$ с $\Omega_{2}=\Omega_{1} e^{i \theta}$, ограниченная, однозначная и $C^{2}$-гладкая всюду вне омбилических точек. В силу выполнения уравнений $(3),(4)$ имеем соотношение

$$
\frac{\partial \Omega_{2}}{\partial \bar{\zeta}}=f=f e^{i \theta}+i \Omega_{1} e^{i \theta} \theta_{\bar{\zeta}}
$$

Умножим это равенство на $-i \bar{\Omega}_{1} e^{-i \theta}$. Получим

$$
g \theta_{\bar{\zeta}}=-i f\left(e^{-i \theta}-1\right) \bar{\Omega}_{1}(=-i f \bar{\varphi})
$$

Запишем сопряженное к (67) выражение:

$$
g \theta_{\zeta}=i \bar{f}\left(e^{i \theta}-1\right) \Omega_{1}
$$

Продифференцируем (67) по $\frac{\partial}{\partial \zeta}$ и, умножив результат на $g$, с учетом уравнений (67), (68) придем к соотношению

$$
g^{2} \theta_{\zeta \bar{\zeta}}=-i\left(e^{-i \theta}-1\right) A \bar{\Omega}_{1}=0
$$

Следовательно, функция $\theta$ гармоническая и омбилические точки являются для нее изолированными особыми точками. В силу ограниченности она продолжается в эти точки как регулярная гармоническая функция и поэтому на компактной поверхности без края она может быть только константой. А тогда по равенству (67) получаем $f=0$, т.е. $H=$ const.

ЗАмЕчАниЕ 12. Отсутствие непрерывного семейства Бонне компактных поверхностей является хорошо известным результатом (см., например, [9]), но 
мы здесь утверждаем, что отсутствуют и дискретные пары Бонне (в принципе этот факт не следует из отсутствия непрерывного семейства, например неизгибаемая поверхность может иметь дискретное множество изометричных ей поверхностей). К тому же при условиях теоремы не известно, отсутствуют ли непрерывные семейства Бонне, так как для локального существования семейства Бонне необходимо выполнение соотношения (49), а следует ли оно из наличия равенства $A=0$, не известно (см. замечание 10$)$.

ЗАмечАНИЕ 13. На самом деле из доказательства теоремы 13 следует немного больше, чем написано в ее формулировке, так как фактически мы доказали, что на римановом многообразии $V$ нет двух дифференциалов Хопфа, определяемых данными формами $f$ и $g$. А могло случиться, что есть две формы и одна данная поверхность, а второй поверхности нет, так как для топологически сложных областей наличие двух согласованных уравнениями ПетерсонаКодацци кандидатов в основные формы поверхности еще не означает существование двух соответствующих им поверхностей.

ЗАмечАниЕ 14. Для получения аналогичного теореме 13 результата в меньшем классе гладкости (а именно, в $C^{3}$ ) достаточно предположить, что имеет место представление (49).

Рассмотрим теперь случай, когда на поверхности $S$ есть точки, в которых $A \neq 0$. В этих точках коэффициент $\Omega$ дифференциала Хопфа поверхности согласно (23) представляется в виде

$$
\Omega(\zeta)=\frac{B+i \varepsilon \sqrt{\Delta}}{2 \bar{A}}
$$

где $\Delta=4 g A \bar{A}-B^{2} \geqslant 0, \varepsilon= \pm 1$. Учитывая закон изменения всех участвующих в формуле (70) величин при конформной замене координат, убеждаемся, что значение $\varepsilon$ не зависит от выбора локальной карты. Отметим, что в точках с $A=0$ должно быть $\Delta=0$.

ТеОрема 14. Пусть на $C^{4}$-гладкой компактной поверхности во всех точках с $A \neq 0$ в формуле (70) коэфбициент в имеет одинаковый знак. Тогда для этой поверхности нет пары Бонне.

ДокАзАтЕльство. С учетом формулы (70) равенство (69) можно переписать так:

$$
g^{2} \theta_{\zeta \bar{\zeta}}=\sin \frac{\theta}{2}\left(\varepsilon \sqrt{\Delta} \sin \frac{\theta}{2}-B \cos \frac{\theta}{2}+i\left(B \sin \frac{\theta}{2}+\epsilon \sqrt{\Delta} \cos \frac{\theta}{2}\right)\right) .
$$

Мнимая часть справа равна нулю, поэтому имеем

$$
B=-\frac{\epsilon \sqrt{\Delta} \cos \frac{\theta}{2}}{\sin \frac{\theta}{2}}
$$

причем на всей поверхности $\sin \frac{\theta}{2} \neq 0$ (в омбилических точках имеются в виду предельные значения $\theta$ ). Подставляя это значение $B$ в $(71)$, получаем

$$
g^{2} \theta_{\zeta \bar{\zeta}}=\epsilon \sqrt{\Delta}
$$


В силу предположения теоремы в точках, где $A \neq 0$, правая часть имеет постоянный знак, а в точках с $A=0$ имеем $\Delta=0$. В таком случае $\theta$ оказывается ограниченной субгармонической (или супергармонической) функцией с изолированными особенностями в омбилических точках. Известно, что такая функция продолжима в изолированные особые точки, оставаясь в своем классе (см. [25; гл. 5]). Функция ограничена сверху (снизу) на всей поверхности (например, числом $2 \pi$ сверху и числом 0 снизу), поэтому существует число $C=\sup \theta$. Используя определение точной верхней грани и компактность поверхности, получаем, что на поверхности найдется сходящаяся последовательность точек $M_{1}, M_{2}, \ldots, M_{n} \rightarrow M_{0}, n \rightarrow \infty$, по которой последовательность $\theta\left(M_{n}\right)$ стремится к $C$. Но по принципу максимума субгармонических функций такая точка является граничной точкой области или же функция должна быть постоянной (аналогичное рассуждение проводим с inf $\theta$ в случае супергармонической функции). Граничных точек нет, значит, $\theta(\zeta) \equiv$ const, что и доказывает теорему.

Отметим, что условие теоремы 14 гарантированно выполняется, если множество точек с $A=0$ не разбивает поверхность $S$ на несвязные компоненты, или, что то же самое, множество $S /\{x \in S \mid A(x)=0\}$ связно.

Рассмотрим далее случай, когда на множестве точек с $A \neq 0$ в формуле (70) могут встречаться $\varepsilon$ с разными знаками. В этом случае удается указать другие функции, являющиеся при определенных формальных условиях субили супергармоническими, и, тем самым, опять получить новые достаточные условия для отсутствия пары Бонне. Приведем примеры таких функций.

УТВЕРЖДЕНИЕ 8. При выполнении условия $B+g f \bar{f} \geqslant 0$ или $B+g f \bar{f} \leqslant 0$ поверхность не имеет пары Бонне.

ДоказАТЕЛЬСтво. Имеет место равенство

$$
\frac{1}{2}\left(\cos \frac{\theta}{2}\right) \theta_{\zeta \bar{\zeta}}=\left(\sin \frac{\theta}{2}\right)_{\zeta \bar{\zeta}}+\frac{1}{4} \sin \frac{\theta}{2} \theta_{\zeta} \theta_{\bar{\zeta}}
$$

Из уравнений (72) и (73) с учетом (74) получаем уравнение

$$
B=-\frac{2 g^{2}}{\sin \frac{\theta}{2}}\left[\left(\sin \frac{\theta}{2}\right)_{\zeta \bar{\zeta}}+\frac{1}{4} \sin \frac{\theta}{2} \theta_{\zeta} \theta_{\bar{\zeta}}\right] .
$$

В то же время непосредственными вычислениями получаем

$$
-2 g^{2}\left(\ln \sin \frac{\theta}{2}\right)_{\zeta \bar{\zeta}}=-2 g^{2}\left[\frac{\left(\sin \frac{\theta}{2}\right)_{\zeta \bar{\zeta}}}{\sin \frac{\theta}{2}}-\frac{\cos ^{2} \frac{\theta}{2}}{4 \sin ^{2} \frac{\theta}{2}} \theta_{\zeta} \theta_{\bar{\zeta}}\right] .
$$

Подставляя сюда значение $\left(\sin \frac{\theta}{2}\right)_{\zeta \bar{\zeta}}$ из $(75)$ и учитывая формулы $(67),(68)$ и (13), получаем уравнение

$$
-2 g^{2}\left(\ln \sin \frac{\theta}{2}\right)_{\zeta \bar{\zeta}}=B+2 g f \bar{f},
$$

откуда в силу суб- или супергармоничности функции $\ln \sin \frac{\theta}{2}$ на компактной поверхности и следует высказанное утверждение. Из уравнения (76) с учетом 
двух форм уравнения для $B$ в (18) получаем следующие необходимые условия для существования пары Бонне.

СЛЕДСТвИЕ 7. Если поверхность $S$ допускает пару Бонне, то верно интегральное равенство

$$
\frac{1}{2 i} \sum \oint \frac{\partial}{\partial \zeta} \ln \left(H^{2}-K\right) d \zeta=\iint_{S} K d S,
$$

где сумма берется по ииклам, окружающим омбилические точки с обходом, оставляющим окрестности этих точек слева.

Отметим, что это равенство дает новое доказательство установленного в начале параграфа соотношения $N=4 p-4$, где $N$ - число нулей голоморфного дифференциала $\varphi$, а $p$ - род поверхности.

СлеДСтвиЕ 8. Если для поверхности $S$ существует пара Бонне, то справедливо интегральное равенство

$$
\iint_{S}\left(\frac{1}{g \Lambda^{2}} \frac{\partial^{2} g}{\partial \zeta \partial \bar{\zeta}}-\frac{1}{g^{2} \Lambda^{2}} \frac{\partial g}{\partial \zeta} \frac{\partial g}{\partial \bar{\zeta}}\right) d S=\iint_{S} \frac{B+2 g f \bar{f}}{g^{2} \Lambda^{2}} d S=0 .
$$

Значит, утверждение 8 о несуществовании пары Бонне справедливо при более слабом условии

$$
\iint_{S} \frac{B+2 g f \bar{f}}{g^{2} \Lambda^{2}} d S \neq 0
$$

СлЕДСтвиЕ 9. Если на поверхности всюду $B \equiv 0$, то она не имеет пару Бонне.

Уравнение (75) позволяет получить большое количество формально необходимых условий существования пары Бонне в интегральной форме с использованием функций вида $G=F\left(\sin \frac{\theta}{2}\right)$, где $F$ - произвольная $C^{2}$-гладкая функция на поверхности, для чего достаточно выразить лапласиан функции $G$ через лапласиан функции $\sin \frac{\theta}{2}$. Приведем примеры таких функций. Рассмотрим функции $G_{a}=\sin ^{a} \frac{\theta}{2}$, где $a-$ любое действительное число. Прямыми вычислениями с использованием формул $(13),(67)$ и (72) получаем

$$
\left(\sin ^{a} \frac{\theta}{2}\right)_{\zeta \bar{\zeta}}=-\frac{a \sin ^{a} \frac{\theta}{2}}{2 g^{2}}\left(B+2 g f \bar{f}-2 a g f \bar{f} \cos ^{2} \frac{\theta}{2}\right) .
$$

Из этого равенства интегрированием по всей поверхности получаем соотношение

$$
\iint_{S} \frac{(B+2 g f \bar{f}) \sin ^{a} \frac{\theta}{2}}{g^{2} \Lambda^{2}} d S=2 a \iint_{S} \frac{g f \bar{f} \cos ^{2} \frac{\theta}{2} \sin ^{a} \frac{\theta}{2}}{g^{2} \Lambda^{2}} d S
$$

(в точках, где $g=0$, существование интегралов следует из соотношений $\frac{B}{g^{2}}=$ $\left.O\left(\left|\theta_{\zeta \bar{\zeta}}\right|\right), \frac{f \bar{f}}{g}=O\left(\left|\theta_{\zeta}\right|^{2}\right)\right)$. Интеграл в правой части равенства (77) всегда положителен, а левая его часть имеет тот же знак, что и параметр а. Далее, на обе части этого равенства можно смотреть как на аналитические функции от параметра $а$ с распространением его на комплексную плоскость. Тогда разложением 
в окрестности нуля по степеням $a$ получаем такие значения его коэффициентов Тейлора $a_{k}$ при степени $a^{k}, k \geqslant 1\left(a_{0}=0\right)$ :

$$
a_{k}=\frac{1}{k !} \iint_{S} \frac{B+2 g f \bar{f}}{g^{2} \Lambda^{2}} \ln ^{k} \sin \frac{\theta}{2} d S=\frac{2}{(k-1) !} \iint_{S} \frac{g f \bar{f} \cos ^{2} \frac{\theta}{2}}{g^{2} \Lambda^{2}} \ln ^{k-1} \sin \frac{\theta}{2} d S
$$

причем знак коэффициента $a_{k}$ в левой части равен $(-1)^{k-1}$. Отметим, что выполнение всех этих равенств является необходимым условием существования пары Бонне для данной поверхности $S$, так как для нее значения $\sin \frac{\theta}{2}$ и $\cos \frac{\theta}{2}$ вычисляются по метрике и средней кривизне самой поверхности по формулам

$$
\sin \frac{\theta}{2}=\sqrt{1-\frac{B^{2}}{4 g A \bar{A}}}, \quad \cos ^{2} \frac{\theta}{2}=\frac{B^{2}}{4 g A \bar{A}}, \quad \text { где } \quad \frac{B^{2}}{4 g A \bar{A}}<1 \quad \text { на } S .
$$

Все приведенные выше интегральные формулы были внутренними характеристиками пары Бонне, так как они выражали условия существования на многообразии $V$ дифференциалов Хопфа. Но существование двух дифференциалов Хопфа только необходимо, а вовсе еще не достаточно для существования пары поверхностей Бонне. Рассмотрим теперь интегральные равенства, связанные уже с предположением существования самих поверхностей Бонне. Допустим, есть пара Бонне. Обратимся тогда к формуле (65). Для пары Бонне первые два интеграла в ее левой части исчезают. С учетом равенств

$M-M^{*}=\frac{1}{2} \operatorname{Im} \varphi, \quad L-L^{*}=-\frac{1}{2} \operatorname{Re} \varphi, \quad N-N^{*}=\frac{1}{2} \operatorname{Re} \varphi, \quad \varphi=\Omega^{*}-\Omega$ и с применением операций $\frac{\partial}{\partial \zeta}$ и $\frac{\partial}{\partial \bar{\zeta}}$ мы можем переписать ее в виде

$$
\iint_{S} p h \frac{\varphi \bar{\varphi}}{4 \Lambda^{4}} d S=\iint_{S} \frac{\left(\mathbf{r}, \mathbf{r}_{\bar{\zeta}}\right) \varphi h_{\bar{\zeta}}+\left(\mathbf{r}, \mathbf{r}_{\zeta}\right) \bar{\varphi} h_{\zeta}}{\Lambda^{4}} d S
$$

Положим $h=1$. Получим

$$
\iint_{S} p \frac{\varphi \bar{\varphi}}{4 \Lambda^{4}} d S=0
$$

где, напомним, $p=(\mathbf{r}, \mathbf{n})$ - опорная функция поверхности, а $\mathbf{n}-$ единичная нормаль. Так как $\varphi \bar{\varphi}$ по формуле (13) выражается через $g$ и $\theta$, равенство (81) дает для поверхности $S$ необходимое интегральное условие существования ее пары Бонне

$$
\iint_{S} p\left[4\left(H^{2}-K\right)-\frac{B^{2}}{4 A \bar{A} \Lambda^{4}}\right] d S=0
$$

где все величины выражаются через радиус-вектор данной поверхности. С использованием формул (13) и (79) равенство (82) может быть представлено в более компактном виде

$$
\iint_{S} p\left(H^{2}-K\right) \sin ^{2} \frac{\theta}{2} d S=0
$$


Далее, рассмотрим интеграл

$$
J=\iint_{S} \frac{\left(\mathbf{r r}_{\bar{\zeta}}\right) \Phi h_{\bar{\zeta}}}{\Lambda^{4}} d S
$$

где $\Phi$ - коэффициент произвольного квадратичного голоморфного дифференциала, $h$ - произвольная $C^{1}$-гладкая функция на поверхности. Этот интеграл можно преобразовать следующим образом:

$$
J=\iint_{S}\left(\frac{\left(\mathbf{r r}_{\bar{\zeta}}\right) \Phi h}{\Lambda^{2}}\right)_{\bar{\zeta}} d \xi d \eta-\iint_{S} h \Phi \frac{\left(\mathbf{r r}_{\bar{\zeta} \bar{\zeta}}\right) \Lambda-2 \Lambda_{\bar{\zeta}}\left(\mathbf{r r}_{\bar{\zeta}}\right)}{\Lambda^{3}} d \xi d \eta=-\iint_{S} \frac{p h \Phi \bar{\Omega}}{4 \Lambda^{4}} d S
$$

что дает уравнение

$$
\iint_{S} \frac{\left(\mathbf{r r}_{\bar{\zeta}}\right) \Phi h_{\bar{\zeta}}}{\Lambda^{4}} d S=-\iint_{S} \frac{p h \Phi \bar{\Omega}}{4 \Lambda^{4}} d S
$$

(при получении этого уравнения учтены деривационное соотношение $4 \Lambda \mathbf{r}_{\zeta \bar{\zeta}}=$ $8 \Lambda_{\bar{\zeta}} \mathbf{r}_{\bar{\zeta}}+\bar{\Omega} \mathbf{n}$ и равенство $\mathbf{r}_{\bar{\zeta}}^{2}=0$, которое выполняется в силу изотермичности системы внутренних координат).

Отметим, что уравнение (84) имеет место для любой компактной поверхности (а не только для поверхности из пары Бонне) и коэффициента $\Phi$ любого голоморфного квадратичного дифференциала. В случае выбора $\Phi=\varphi$ сложение уравнения (84) с ему сопряженным дает уравнение (80), так как тогда $\varphi \bar{\Omega}+\bar{\varphi} \Omega=-\varphi \bar{\varphi}$.

Положим в (84) $h=1$ и получим

$$
\iint_{S} \frac{p \Phi \bar{\Omega}}{\Lambda^{4}} d S=0
$$

Выберем теперь $\Phi=\varphi$. Тогда с учетом формулы (11) получим

$$
\frac{1}{2} \iint_{S} \frac{p \varphi \bar{\varphi}}{\Lambda^{4}} d S+\frac{i}{2} \iint_{S} \frac{p \varphi \bar{\varphi} \cot \frac{\theta}{2}}{\Lambda^{4}} d S=0
$$

Действительная часть этого равенства дает уже известное нам соотношение (81), а мнимая часть дает новое уравнение

$$
\iint_{S} p\left(H^{2}-K\right) \sin \theta d S=0 .
$$

Формула (81) верна при всех положениях поверхности в пространстве. Сдвинем ее на произвольный вектор С. Из равенства (81) получим

$$
\iint_{S}(\mathbf{C n}) \frac{\varphi \bar{\varphi}}{4 \Lambda^{4}} d S=0,
$$

откуда в силу произвольности $\mathbf{C}$ получаем равенство

$$
\iint_{S} \frac{\varphi \bar{\varphi}}{4 \Lambda^{4}} \mathbf{n} d S=0 .
$$


Аналогично, соотношения (83), (85) и (86) дают соответственно формулы

$$
\begin{gathered}
\iint_{S}\left(H^{2}-K\right) \sin ^{2} \frac{\theta}{2} \mathbf{n} d S=0, \quad \iint_{S} \frac{\Phi \bar{\Omega}}{\Lambda^{4}} \mathbf{n} d S=0, \\
\iint_{S}\left(H^{2}-K\right) \sin \theta \mathbf{n} d S=0 .
\end{gathered}
$$

Таким образом, мы получили ряд интегральных равенств, выполнение которых необходимо для существования пары Бонне для данной поверхности. Стоит заметить, что эти условия реально отсеивают некоторые поверхности из числа могущих иметь свою пару Бонне ${ }^{17}$, но мы не выяснили, насколько широк класс поверхностей, попадающих под действие наших интегральных условий. $\mathrm{K}$ тому же потенциал формул (80) и (84) для выявления новых необходимых интегральных условий для пар Бонне представляется еще далеко не исчерпанным.

ЗАмечАниЕ 15. Коротко скажем о проблеме пар Бонне в случае поверхностей с постоянной средней кривизной. Из формулы

$$
2 \mathbf{n}_{\bar{\zeta}}=-2 H \mathbf{r}_{\bar{\zeta}}-\frac{\bar{\Omega}}{\Lambda^{2}} \mathbf{r}_{\zeta}
$$

получаем следующее уравнение для нормали:

$$
\Delta \mathbf{n}+2\left(2 H^{2}-K\right) \Lambda^{2} \mathbf{n}+4 \operatorname{Re}\left(H_{\zeta} \mathbf{r}_{\bar{\zeta}}\right)=0,
$$

которое в случае постоянной кривизны превращается в уравнение для спектра оператора Лапласа-Бельтрами $\widetilde{\Delta}$

$$
\widetilde{\Delta} \mathbf{n}+2\left(2 H^{2}-K\right) \mathbf{n}=0
$$

со строго положительным коэффициентом при неизвестной вектор-функции $\mathbf{n}$. Известно, что такие однородные уравнения на компактных многообразиях имеют только конечномерное множество решений (три решения уже есть - это три компоненты нормали), следовательно, компактные поверхности с постоянной средней кривизной не могут изгибаться с сохранением средней кривизны, а число парных по Бонне поверхностей может быть только конечным. Таким образом, появляется задача определения числа таких поверхностей в функции от различных их характеристик.

\section{$\S 13$. Заключение}

В конце позволим себе привести некоторый комментарий исторического хаpaктера. Формула (23) была получена нами в феврале 2004 г. во время стажировки во Франции в IHES. Однако впоследствии (по информации Р. Суама из

\footnotetext{
${ }^{17}$ Например, по условию (81) у звездных поверхностей не может быть пары Бонне, хотя этот факт, конечно, не дает нам новый класс поверхностей без пары Бонне.
} 
университета Париж-7, которому я здесь приношу свою искреннюю благодарность) я познакомился с некоторыми не известными мне работами по проблеме Бонне о единственности поверхности с заданными метрикой и средней кривизной. В одной из этих работ я обнаружил ссылку на работу Томаса 1945 г. [26], в которой, оказывается, уже была получена аналогичная (23) формула даже в случае метрики общего вида, а поэтому более сложными рассуждениями, чем у нас. Соответственно, формулы Томаса имеют более сложный и менее удобный для применений вид.

\section{Список литературы}

[1] O. Bonnet, "Mémoire sur la théorie des surfaces applicables", J.École Polytechnique, 42 (1867), 72-92.

[2] É. Cartan, "Sur les couples de surfaces applicables avec conservation des courbures principales", Bull. Sci. Math. (2), 66 (1942), 55-72, 74-85.

[3] К. М. Белов, "Изгибание поверхностей с сохранением средней кривизны", Сиб. матем. журн., 9:1 (1986), 194-198; англ. пер.: K. M. Belov, "Bending of surfaces with retention of mean curvature", Siberian Math. J., 9:1 (1968), 146-149.

[4] Sh.-Sh. Chern, "Deformation of surfaces preserving principal curvatures", Differential geometry and complex analysis, Springer, Berlin, 1985, 155-163.

[5] X. Chen, Ch.-K. Peng, "Deformation of surfaces preserving principal curvatures", Differential geometry and topology (Tianjin, 1986-1987), Lecture Notes of Math., 1369, Springer-Verlag, Berlin, 1989, 63-70.

[6] G. I. Kamberov, "Quadratic differentials and surface shape", Rend. Semin. Mat. Messina, Ser. II Suppl., 2000, 199-210.

[7] G. Kamberov, F. Pedit, U. Pinkall, "Bonnet pairs and isothermic surfaces", Duke Math. J., 92:3 (1998), 637-644.

[8] A.I. Bobenko, "Exploring surfaces through methods from the theory of integrable systems: the Bonnet problem", Surveys on geometry and integrable systems (Tokyo, Japan, 2000), Adv. Stud. Pure Math., 51, Math. Soc. Japan, Tokyo, 2008, 1-53.

[9] A. I. Bobenko, U. Eitner, Painlevé equations in the differential geometry of surfaces, Lecture Notes in Math., 1753, Springer-Verlag, Berlin, 2001.

[10] R. Tribuzy, "A characterization of tori with constant mean curvature in space form", Bull. of Braz. Math. Soc., 11:2 (1980), 259-274.

[11] H. B. Lawson, jr., R. Tribuzy, "On the mean curvature function for compact surfaces", J. Differential Geom., 16:1 (1981), 179-183.

[12] И.Н. Векуа, Обобщенные аналитические функиии, Наука, М., 1959; англ. пер.: I. N. Vekua, Generalized analytic functions, Pergamon, London-Paris-Frankfurt; Addison-Wesley, Reading, MA, 1962.

[13] Ф. Д. Гахов, "Краевые задачи", 1977; англ. пер. 2-го изд.: F. D. Gakhov, Boundary value problems, 3-е изд., Pergamon, London-Paris-Frankfurt; Addison-Wesley, Reading, MA, 1966.

[14] Ph. Hartman, A. Wintner, "Gaussian curvature and local embedding", Amer. J. Math., 73:4 (1951), 876-884.

[15] И. Х. Сабитов, С. З. Шефель, "О связях между порядками гладкости поверхности и ее метрики”, Сиб. матем. журн., 17:4 (1976), 916-925; англ. пер.: I. Kh. Sabitov, S.Z. Shefel', "The connections between the order of smoothness of a surface and its metric", Siberian Math. J., 17:4 (1976), 687-694. 
[16] И.Х. Сабитов, “Двумерные многообразия с метриками вращения”, Матем. сб., 191:10 (2000), 87-104; англ. пер.: I. Kh. Sabitov, "Two-dimensional manifolds with metrics of revolution", Sb. Math., 191:10 (2000), 1507-1525.

[17] R. Alexander, "Lipschitzian mappings and total mean curvature of polyhedral surfaces. I", Trans. Amer. Math. Soc., 288:2 (1985), 661-678.

[18] F. J. Almgren, jr., I. Rivin, "The mean curvature integral is invariant under bending", The Epstein birthday schrift, Geom. Topol. Monogr., 1, Univ. of Warwick, Warwick, 1998, 1-21.

[19] J.-M. Schlenker, R. Souam, "Higher Schläfli formulas and applications", Compositio Math., 135, 2003, 1-24.

[20] В.А. Александров, "Об интегральной средней кривизне нежестких поверхностей", Сиб. матем. журн., 50:5 (2009), 963-966; англ. пер.: V. A. Alexandrov, "On the total mean curvature of a nonrigid surface", Siberian Math. J., 50:5 (2009), 757-759.

[21] I. Kh. Sabitov, "Some integral formulas for compact surfaces", TWMS J. Pure Appl. Math., 1:1 (2010), 123-131.

[22] Н. В. Ефимов, "Качественные вопросы теории деформаций поверхностей", УМН, 3:2 (1948), 47-158.

[23] А. Гурвиц, Р. Курант, Теория функиий, Наука, М., 1968; пер. с нем.: A. Hurwitz, Vorlesungen über allgemeine Funktionentheorie und elliptische Funktionen, SpringerVerlag, Berlin-New York, 1964.

[24] И. Х. Сабитов, "Решение проблемы пар Бонне", Докл. РАН, 434:2 (2010), 164-167; англ. пер.: I. Kh. Sabitov, "A solution of the Bonnet pairs problem", Dokl. Math., 82:2 (2010), 722-725.

[25] У. Хейман, П. Кеннеди, Субгармонические функиии, Мир, М., 1980; пер. с англ.: W. K. Hayman, P. B. Kennedy, Subharmonic functions, London Math. Soc. Monogr. Ser., 9, Academic Press, London-New York-San Francisco, 1976.

[26] T. Y. Thomas, "Algebraic determination of the second fundamental form of a surface by its mean curvature", Bull. Amer. Math. Soc., 51 (1945), 390-399.

И. Х. Сабитов (I. Kh. Sabitov)

Механико-математический факультет

Московского государственного университета

им. М. В. Ломоносова

E-mail: isabitov@mail.ru
Поступила в редакцию 09.03.2010 и 09.04.2011 